\title{
The globular cluster systems of 54 Coma ultra-diffuse galaxies: statistical constraints from $H S T$ data
}

\author{
N. C. Amorisco ${ }^{1,2 \star}$, A. Monachesi ${ }^{1,3,4}$, A. Agnello ${ }^{5}$, S. D. M. White ${ }^{1}$ \\ ${ }^{1}$ Max Planck Institute for Astrophysics, Karl-Schwarzschild-Strasse 1, 85748 Garching, Germany \\ 2 Institute for Theory and Computation, Harvard-Smithsonian centre for Astrophysics, 60 Garden St., Cambridge, MA 02138, USA \\ ${ }^{3}$ Instituto de Investigacion Multidisciplinario en Ciencia y Tecnologia, Universidad de La Serena, Raul Bitran 1305, La Serena, Chile \\ ${ }^{4}$ Departamento de Fisica y Astronomia, Universidad de La Serena, Av. Juan Cisternas 1200 N, La Serena, Chile \\ ${ }^{5}$ European Southern Observatory, Karl-Schwarzschild-Strasse 2, 85748 Garching, Germany
}

12 January 2018

\begin{abstract}
We use data from the HST Coma Cluster Treasury program to assess the richness of the Globular Cluster Systems (GCSs) of 54 Coma ultra-diffuse galaxies (UDGs), 18 of which have a half-light radius exceeding $1.5 \mathrm{kpc}$. We use a hierarchical Bayesian method tested on a large number of mock datasets to account consistently for the high and spatially varying background counts in Coma. These include both background galaxies and intra-cluster GCs (ICGCs), which are disentangled from the population of member GCs in a probabilistic fashion. We find no candidate for a GCS as rich as that of the Milky Way, our sample has GCSs typical of dwarf galaxies. For the standard relation between GCS richness and halo mass 33 galaxies have a virial mass $M_{v i r} \leqslant 10^{11} M_{\odot}$ at $90 \%$ probability. Only three have $M_{v i r}>10^{11} M_{\odot}$ with the same confidence. The mean colour and spread in colour of the UDG GCs are indistinguishable from those of the abundant population of ICGCs. The majority of UDGs in our sample are consistent with the relation between stellar mass and GC richness of 'normal' dwarf galaxies. Nine systems, however, display GCSs that are richer by a factor of 3 or more (at $90 \%$ probability). Six of these have sizes $\lesssim 1.4 \mathrm{kpc}$. Our results imply that the physical mechanisms responsible for the extended size of the UDGs and for the enhanced GC richness of some cluster dwarfs are at most weakly correlated.
\end{abstract}

Key words: galaxies: dwarf - galaxies: structure — galaxies: formation — galaxies: haloes — galaxies: clusters

\section{INTRODUCTION}

Ultra-diffuse galaxies (UDGs) are a population of lowsurface brightness systems (effective surface brightness $\langle\mu\rangle_{r} \gtrsim 24 \mathrm{mag} / \operatorname{arcsec}^{2}$ ) with stellar masses typical of dwarf galaxies $\left(7 \lesssim \log M_{*} / M_{\odot} \lesssim 9\right)$. Ubiquitous in nearby galaxy clusters (van Dokkum et al. 2015; Koda et al. 2015; Muñoz et al. 2015; van der Burg et al. 2016; Mihos et al. 2015; Venhola et al. 2017; Lee et al. 2017), UDGs have also been found outside cluster environments (Martínez-Delgado et al. 2016; Román \& Trujillo 2017; Trujillo et al. 2017; Bellazzini et al. 2017; Leisman et al. 2017; Shi et al. 2017; Greco et al. 2017). In clusters, they appear as roundish featureless spheroids (e.g. Yagi et al. 2016; Mowla et al. 2017), which extend the red sequence of cluster galaxies in the colourmagnitude diagram into the regime of dwarf galaxies (Koda et al. 2015; van der Burg et al. 2016, 2017; Gu et al. 2017),

^ E-mail: nicola.amorisco@cfa.harvard.edu with hints of a trend to bluer colours in less dense environments (Román \& Trujillo 2017).

UDGs are certainly highly dark matter dominated systems (van Dokkum et al. 2015; Beasley et al. 2016; Amorisco \& Loeb 2016; van Dokkum et al. 2016), and particular interest has been sparked by the mismatch between their luminosity and their sizes, prompting the proposal that their halo mass could be much larger than suggested by their stellar mass (van Dokkum et al. 2015; Koda et al. 2015; van Dokkum et al. 2016). Within this scenario, UDGs are prematurely quenched galaxies, which 'fail' to form their stars because of their early infall onto the galaxy cluster (Yozin \& Bekki 2015; van Dokkum et al. 2015). The largest UDGs (e.g. half-light radii $\gtrsim 1.5 \mathrm{kpc}$ ) could then be hosted by Milky Way (MW) mass haloes rather than by haloes with masses below or similar to that of the Large Magellanic Cloud (LMC).

One clue to the nature of UDGs is the almost perfect linearity of the relation between the abundance of UDGs in

(c) 0000 The Authors 

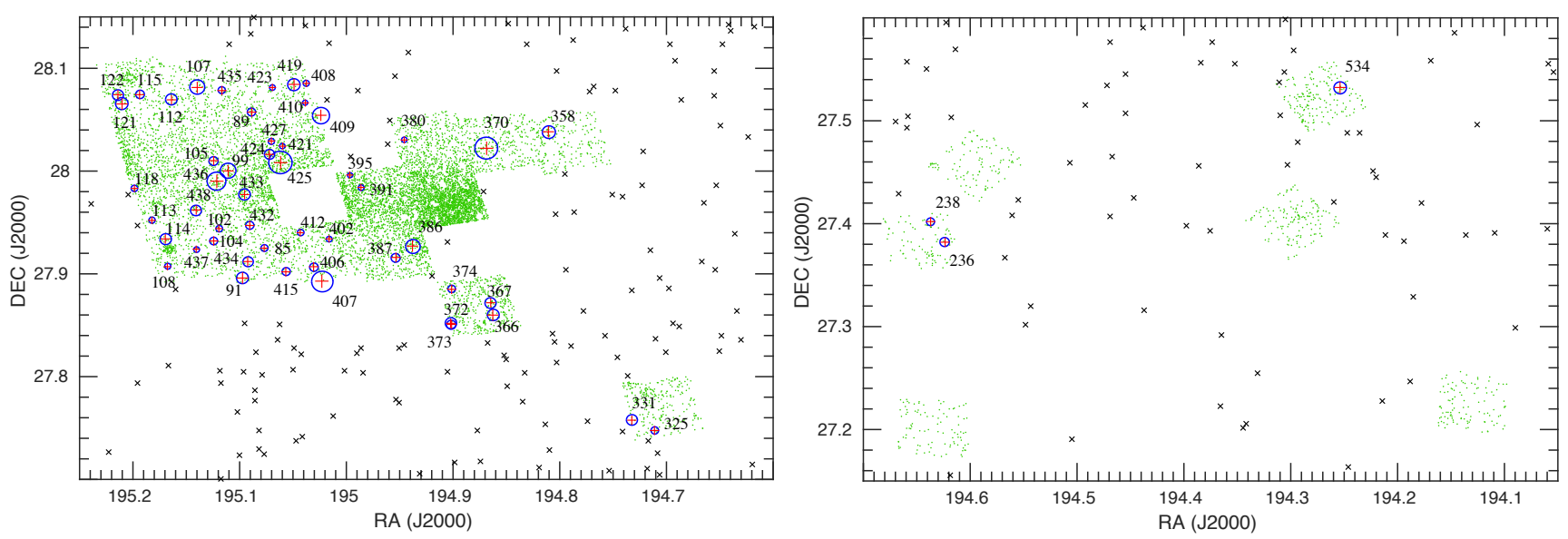

Figure 1. A composite of the HST/ACS fields observed as part of the Coma Cluster Treasury program, together with the Coma UDGs from Yagi et al. (2016), shown as black and red crosses. Green points are candidate GCs selected from the Hammer et al. (2010) catalogue, selected as in Peng et al. (2011). The 54 UDGs whose centres fall within the observed HST fields are marked in red, their size is shown by a blue circle (with a radius of $6 \times R_{S}$ ), and their ID number in the Yagi et al. (2016) catalogue is displayed.

clusters and the cluster mass itself (van der Burg et al. 2016, 2017; Janssens et al. 2017). Within the LCDM framework, a linear relation is expected if the formation mechanism of UDGs is independent of environment. As the shape of the subhalo mass function is independent of the virial mass of the central (e.g., Gao et al. 2004; Giocoli et al. 2008), a linear relation emerges naturally if UDGs are just a fraction of the cluster subhalo population with the relevant mass. This suggests that the physical mechanism that gives UDGs their unusual properties has an 'internal' origin, and, in contrast with the scenario above, that it is unrelated to the interaction with the cluster environment. This is corroborated by the detection of UDGs outside cluster cores (MartínezDelgado et al. 2016; Román \& Trujillo 2017) and in the field (Trujillo et al. 2017; Bellazzini et al. 2017).

If hosted by MW mass haloes, UDGs would lie far from the standard $M_{*}-M_{\text {vir }}$ relation, requiring their formation pathway to differ fundamentally from that of 'normal' haloes of the same total mass. In general, haloes of MW mass appear to be the most efficient at converting gas into stars (e.g. Guo et al. 2010; Behroozi et al. 2013; Moster et al. 2013, and references therein) so the UDG haloes would have to be exceptional objects with very low efficiency. If on the other hand UDG haloes are similar in mass to those of normal dwarf galaxies, their properties could be accommodated by a simple $\Lambda$ CDM framework in which they are just the low surface brightness tail of the abundant population of dwarf galaxies (Amorisco \& Loeb 2016). This picture is consistent with UDGs existing both inside and outside clusters and needs no ad hoc mechanism to make them depart from the $M_{*}-M_{\text {vir }}$ relation. Additionally, if hosted by low mass haloes, stellar feedback during their formation might lead to expansion and so contribute to their large sizes (Di Cintio et al. 2017).

Unfortunately, only a handful of mass measurements are available so far for UDGs. Apart from the recent stacked analysis of Sifón et al. (2017), these are all indirect, based either on the richness of the globular cluster system (GCS), or on extrapolation to the virial radius of a dynamical mass estimated in the main stellar body of the galaxy (Beasley et al. 2016; Beasley \& Trujillo 2016; Peng \& Lim 2016; van Dokkum et al. 2016). Both techniques have their limitations. The approximate linearity of the relation between GCS richness and halo virial mass is supported by a solid pool of evidence, at least for 'normal' galaxies (e.g., Harris et al. 2013; Hudson et al. 2014; Forbes et al. 2016, and references therein), but the mean conversion factor remains uncertain (Harris et al. 2015; Zaritsky et al. 2016; Harris et al. 2017; Georgiev et al. 2010, hereafter G10). Dynamical measurements, however, do not guarantee higher precision, as they can only estimate the mass enclosed in the central regions of the galaxy, where dynamical tracers (stars or GCs) are present. The extrapolation from the galaxy's half-light radius to the virial radius is very substantial (e.g. Walker et al. 2009; Wolf et al. 2010; Amorisco \& Evans 2011; Campbell et al. 2017).

In this paper, we increase the number of virial mass estimates for UDGs by a factor $>3$ using imaging data from the HST Coma Cluster Treasury program to constrain the richness of the GCS of 54 Coma UDGs. In comparison to ground-based data, the high resolution of $H S T /$ ACS data helps significantly in distinguishing candidate GCs from background galaxies (e.g., Peng et al. 2011; Beasley \& Trujillo 2016; Peng \& Lim 2016). However, the high contamination rate by the intracluster population of GCs in Coma and by the abundant population of background galaxies implies that careful statistical analysis is needed to gather reliable constraints. Section 2 describes the data we use for this analysis. Section 3 sets out our hierarchical Bayesian approach, which is tested in Appendix A. Section 4 presents our results, which are discussed in Section 5, where conclusions are laid out.

\section{OBSERVATIONS AND METHODS}

We use the compilation of 854 Coma low surface brightness galaxies presented by Yagi et al. (2016, hereafter Y16), based on Subaru Suprime-Cam archival data analysed in Koda et al. (2015). These are selected to have $\langle\mu\rangle_{R}>24 \mathrm{mag} / \mathrm{arcsec}^{2}$ and a stellar half light radius $\geqslant 0.7 \mathrm{kpc}$. Among these, we 
select those systems whose centres lie within the footprint of the Coma Cluster Treasury program, which we use to explore the properties of their GCSs. There are 54 such galaxies, including 18 with stellar half light radius $\geqslant 1.5 \mathrm{kpc}$, which is the criterion used to define UDGs by van Dokkum et al. (2015). In the following, however, we refer to all of our 54 low surface brightness galaxies as UDGs. Their locations are displayed in Fig. 1, together with their ID numbers in the Y16 catalogue. We adopt half-light radii $R_{S}$ from the single Sersic fits presented by Y16. Where these were not deemed reliable, for example because of light from nearby systems, we adopt the listed values returned by SExtractor.

\subsection{Candidate GCs}

We cross-correlate the position of the Y16 UDGs with the catalogue of the HST/ACS Coma Cluster Treasury program (CCTp) presented by Hammer et al. (2010, hereafter H10). This lists all SExtractor sources detected in the deep $F 814 W$ images, as well as the measurements for the $F 475 \mathrm{~W}$ images. The $F 814 W$-band photometry is $80 \%$ and $50 \%$ complete at 26.8 and 27.3, respectively (H10 and Peng et al. 2011). This defines our completeness function $S_{814}$, for which we adopt the functional form suggested by Salinas et al. (2015, eqn. 3, resulting in $\alpha=1.5$ ). Assuming UDGs have a dwarf-like GC luminosity function (GCLF), CCTp data is 50\% complete at the turnover, $F 814 W=27.33 \mathrm{mag}$, (Beasley \& Trujillo 2016). Additionally, if the spread of the GCLF is also in line with that of dwarf galaxies (Gaussian spread of $1.1 \mathrm{mag}$, G10, Miller \& Lotz 2007; Peng et al. 2009), statistically, $49 \%$ of all member GCs are indeed detected in the CCTp data.

Given its pixel-size (0."05/pixel) HST/ACS imaging is well suited to disentangle GC candidates (GCCs) - which appear as point sources at the distance of Coma - from background galaxies, most of which are resolved. The H10 catalogue flags 'point sources' (FLAGS_OBJ=1), based on photometry at different apertures. However, we find this flag to be unreliable for sources that are close to the UDGs' centres as a consequence of the fact that the galaxy light had not been subtracted prior to the production of the catalogue itself. Because of the UDGs' contribution to the aperture flux, compact objects close to the centre of some UDGs may appear extended and be classified as such in the H10 catalogue. We therefore do not consider this flag and correct the aperture photometry of all sources in the catalogue. We do so explicitly on a source by source basis, by subtracting the flux contributed by each UDG at the source's location, within the considered aperture. For this purpose, we use the UDG Sersic surface brightness profiles measured by Y16 (we take $I_{814} \approx R$ for systems on the red sequence, H10, Koda et al. 2015). We perform an analogous correction to the $F 475 W$ measurements, assuming that all UDGs have the same colour $(F 475 W-F 814 W \approx 0.85$, Koda et al. 2015).

Figure 2 shows the H10 catalogue sources in the plane of the $F 814 W_{1.2}-F 814 W_{4}$ concentration index against the $F 814 W_{4}$ photometry $\left(F 814 W_{1.2}\right.$ and $F 814 W_{4}$ are respectively the 1.2-pixel radius and 4-pixel aperture radius photometries) corrected for the above-mentioned UDG flux contribution. The vertical plume of point sources is clearly visible at $F 814 W_{1.2}-F 814 W_{4} \approx 1.0$, mainly composed of the abundant population of Coma intracluster GCs (ICGCs,
Peng et al. 2011). Red arrows in the same Figure show the effect of the flux correction for individual sources (arrows extend between the properties of each of these sources before and after correction). Only the arrows corresponding to sources that satisfy $0.5<F 814 W_{1.2}-F 814 W_{4}<1.5$ after correction but not before correction are displayed, showing that the contribution of the UDGs' surface brightness may indeed cause some sources in the H10 catalogue to appear unduly extended. This effect is important only for those source that lie close to the UDG centre, at radii $\gtrsim 2 R_{S}$ sources are unaffected. However, this effect cannot be neglected, despite the low surface brightness nature of the UDGs' contribution to the aperture flux. As a consequence, we refrain from selecting GCCs through sharp cuts on the catalogue in either concentration index or in colour. There are a number of reasons why this strategy of accounting for the background flux may not be ideal. These include: i) PSF blur in the ground based Y16 data; ii) the possible bias introduced by any central compact nuclei in the Sersic parameters; iii) the possible presence of neighboring objects that would be masked out in the fitting in Y16. As a result, the accuracy of the corrected aperture photometry of the individual sources may be compromised, but, as we explain in the following section, our analysis does not use these values directly. In fact, a posteriori, we find that this method of subtracting the background is effective and well suited for our purpose. The identification of abundant populations concentrated around the UDG centres gives credence to the adopted estimate of the background. These are found to have concentration indexes that are statistically the same as those of the point sources at large galactocentric distances, which are unaffected by either UDG light or our implementation of the background subtraction. As we will show, our analysis identifies at high confidence a number of systems with rich GCSs. In their central regions, the surface density of sources recognized as member GCs is exceedingly high for any significant fraction of them to be contributed by misclassified extended galaxies. If this was the case, we would detect systematically lower surface densities of extended sources in the rich UDGs. We have made this check and found no evidence for it, which gives additional credence to our background subtraction.

In the following, and as explained in Section 3.1 below, we disentangle background galaxies from GCs in Coma by explicitly modelling both concentration and colour distributions, by taking into account measurement uncertainties on a source by source basis. We only remove those sources with $F 814 W_{4}<22$, to avoid foreground MW stars and saturated pixels (H10).

\section{STATISTICAL ANALYSIS}

As shown by Peng et al. (2011) using these same data and confirmed by Fig. 2, the Coma cluster possesses an abundant population of ICGCs. In addition, many tens of background galaxies are detected in each ACS field. As is readily seen by eye (see our Fig. 1 and Fig. 3 in Peng et al. 2011), the CCTp distribution of GCCs displays clear overdensities at the locations of high surface brightness Coma galaxies. In contrast, and despite our above estimate of the depth of the CCTp data, visual inspection does not always reveal a concentra- 


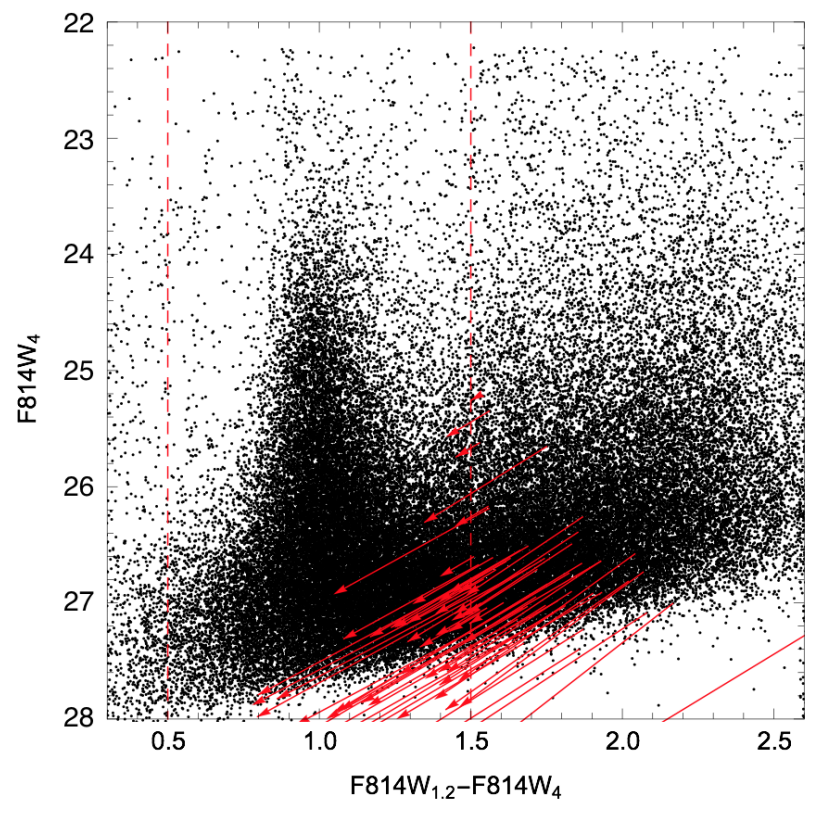

Figure 2. Aperture photometry of sources in the H10 catalogue. The plume of point sources is clearly evident at $F 814 W_{1.2}-$ $F 814 W_{4} \approx 1.0$. Red arrows show those catalogue sources that satisfy $0.5<F 814 W_{1.2}-F 814 W_{4}<1.5$ only after correcting for the UDGs' contribution to the flux within the relevant apertures.

tion of GCCs near the UDG centres. As our analysis will show, the combination of ICGCs and background galaxies is often dominant over the UDGs' GCSs (see Section 4). As a result, we cannot reliably measure their GC abundances simply by counting catalogue sources within some centred aperture and then subtracting an estimate for the background contamination. A more detailed statistical approach, which we describe in the following, is necessary to constrain the richness, $N_{\mathrm{GC}}$ of the GCSs of our UDG sample.

\subsection{The mixture models}

We isolate all H10 sources in the vicinity of each UDG, and model them as the superposition of a slowly spatially varying population of contaminants and a centred population of GCs physically associated with the UDG, often referred to as 'members' in the following. We need to include two different contaminant populations, to include both resolved sources (background galaxies) and contaminants that appear as point sources. The latter population is composed of ICGCs and any remaining foreground stars and unresolved background galaxies that appear as point sources. However, as we show in Section 4, our analysis suggests that the contribution of foreground stars and unresolved background galaxies is negligible, and that this population is indeed dominated by ICGCs. In the absence of close luminous galaxies, we use all catalogue sources that lie within $35 \times R_{S}$ from the UDG's centre. This is a compromise between getting better statistics for the contaminants and modelling their spatial distribution as locally uniform, with surface densities $\Sigma_{I C G C s}$ and $\Sigma_{\text {gal }}$. When near luminous galaxies, we reduce this region on a case by case basis in order to minimise spatially variable contamination (see below and Fig. 3). For convenience, we centre all sources' coordinates on the UDG centre, and rescale them by the stellar half-light radius $R_{S}$. The spatial distribution of the UDG GCS is modelled with a Plummer profile (Plummer 1911)

$\Sigma(R)=\frac{1}{\pi} \frac{1}{R_{h}^{2}\left(1+R^{2} / R_{h}^{2}\right)^{2}}$,

in which $R$ is the projected galactocentric radius and $R_{\mathrm{h}} / R_{S}$, the ratio between the half-number radius $R_{\mathrm{h}}$ and the stellar half-light radius $R_{S}$, is a free parameter, different for each UDG. Experiments using an exponential density profile $\Sigma$ show that the profile shape does not affect our results (see our suite of tests in Appendix A).

As mentioned in Section 2.1 we use both colour

$\mathrm{C} \equiv F 475 W_{1.2}-F 814 W_{1.2}$,

and concentration index

$C_{1.2-4} \equiv F 814 W_{1.2}-F 814 W_{4}$,

to disentangle member GCs from the two contaminant populations. As both member GCs and ICGCs appear as point sources, we assume that both populations have the same distribution in concentration index, which we take to be Gaussian in shape. The mean and dispersion of this Gaussian, $\left\langle C_{1.2-4}\right\rangle_{G C}$ and $\sigma_{G C}\left(C_{1.2-4}\right)$ are free parameters of the model and are fitted for using the corrected H10 catalogue. A different Gaussian probability distribution is used to model the distribution of concentration index of the extended contaminants, with mean $\left\langle C_{1.2-4}\right\rangle_{\text {gal }}$ and dispersion $\sigma_{\text {gal }}\left(C_{1.2-4}\right)$. These 4 free parameters are in fact hyperparameters: they are shared by all of the 54 UDGs in our sample and constraints are determined accordingly, using a hierarchical Bayesian approach. The same is true for the properties of the colour distribution of the extended contaminants, which, for simplicity, we also describe with a Gaussian function, with $\langle\mathrm{C}\rangle_{\text {gal }}$ and dispersion $\sigma_{\text {gal }}(\mathrm{C})$. Instead, the population of ICGCs and the population of member GCs are allowed to have different colour distributions (in both mean and dispersion) on a UDG by UDG basis, so to explore the properties of the UDG GCSs, and because ICGCs may vary in different fields, for example as a function of the UDG's distance from the centre of Coma. Of course, for both colour and concentration index, we do take into account measurement uncertainties on a source by source basis. As we show in Appendix A, use of the magnitude distribution of the sources does not improve the separation of contaminants and member GCs, or the accuracy and precision of the inference on the properties of the UDG GCSs. Given this, we do not use the magnitude distribution in the following analysis.

In conclusion, for the UDG $j$ in our sample, the likelihood of our 3 component model is (e.g., Walker \& Peñarrubia 2011; Amorisco et al. 2014)

$\mathcal{L}_{j}=\prod_{i}^{N_{j}}\left(p_{G C, i}+p_{I C G C, i}+p_{\mathrm{gal}, i}\right)$,

where $i$ runs on the H10 sources in the field defined by the UDG $j$, and, for each source, $p_{X, i}$ is the probability of membership in the population $X$. More explicitly,

$p_{G C, i}=f_{G C, j} \frac{S_{\mathrm{sp}, j}\left(\mathbf{r}_{\mathbf{i}}\right) \Sigma_{j}\left(r_{i}\right)}{\int S_{\mathrm{sp}, j} \Sigma_{j}(r)} \mathcal{G}_{G C}\left(C_{i}\right) \mathcal{G}_{G C s, j}\left(\mathrm{C}_{i}\right)$, 
$p_{I C G C, i}=f_{I C G C, j} \frac{S_{\mathrm{sp}, j}\left(\mathbf{r}_{\mathbf{i}}\right)}{\int S_{\mathrm{sp}, j}} \mathcal{G}_{G C}\left(C_{i}\right) \mathcal{G}_{I C G C s, j}\left(\mathrm{C}_{i}\right)$,

$p_{g a l, i}=\left(1-f_{G C, j}-f_{I C G C, j}\right) \frac{S_{\mathrm{sp}, j}\left(\mathbf{r}_{\mathbf{i}}\right)}{\int S_{\mathrm{sp}, j}} \mathcal{G}_{\mathrm{gal}}\left(C_{i}\right) \mathcal{G}_{\mathrm{gal}}\left(\mathrm{C}_{i}\right)$.

Here,

- $f_{G C, j}$ is the fraction of the total number $N_{j}$ of GCCs in the studied area which are members of the GCS of the UDG $j$. Analogously, the fraction $f_{I C G C, j}$ are ICGCs in the relative field, while the remainder $1-f_{G C, j}-f_{I C G C, j}$ are extended contaminants.

- $S_{\mathrm{sp}, j}(\mathbf{r})$ is the spatial selection function associated with the UDG $j$, whose value is either 0 or 1 . This function accounts for the fact that the area available to study may be limited by the edges of the footprint, or by excised regions surrounding luminous galaxies, in which case its value is 0 . The associated spatial integrals extend out to $35 R_{S}$.

- $C_{i}$ and $\mathrm{C}_{i}$ are the concentration index and colour of the source $i$ and $\mathcal{G}$ is their gaussian distribution. The distribution of the concentration index $\mathcal{G}_{G C}(C)$ is common to all UDGs (and to both GCs and ICGCs), as are $\mathcal{G}_{\text {gal }}(C)$ and $\mathcal{G}_{G C}(\mathrm{C})$. The colour distributions $\mathcal{G}_{G C, j}(\mathrm{C})$ and $\mathcal{G}_{I C G C, j}(\mathrm{C})$ have different mean and spread for each UDG.

In summary, the likelihood $\mathcal{L}_{j}$ involves a total of 7 free parameters per UDG: the dimensionless fractions $f_{G C, j}$ and $f_{I C G C, j}$, the dimensionless ratio $R_{\mathrm{h}} / R_{S}$ between the characteristic radius of the GCS and the UDG's effective radius, mean and spread of the colour distributions of member GCs and ICGCs. In addition, the model includes 6 hyperparameters: mean and spread of the concentration index of point- and extended- sources, mean and spread of the colour distribution of the extended sources. Inference on the latter set is obtained adopting a hierarchical approach, using the model likelihood

$\mathcal{L}=\prod_{j}^{54} \mathcal{L}_{j}$,

which employs data from the entire sample of 54 UDGs at the same time.

Following the tests presented in Appendix A our priors are defined as:

- uniform in $\log f$ for both $f_{G C}$ and $f_{I C G C}$, with the constraint that $f_{G C}+f_{I C G C}<1$; in addition, we require $\log f_{G C}>\log f_{0}-1.5$, where the value of $f_{0}$ is estimated based on the expectation that the UDG has a 'normal' GC abundance for its stellar mass (see Appendix A2 for details), and $\log f_{I C G C}>\log f_{1}-1.5$, where $f_{1}$ corresponds to a total of 1 member of the ICGC population in the whole field;

- uniform in $\log R_{\mathrm{h}} / R_{S}$, in the interval $0.75<R_{\mathrm{h}} / R_{S}<$ 3.5

- uniform in $\langle\mathrm{C}\rangle$ in the interval $0<\langle\mathrm{C}\rangle<3$ for all of $\langle\mathrm{C}\rangle_{G C},\langle\mathrm{C}\rangle_{I C G C},\langle\mathrm{C}\rangle_{\text {gal }}$;

- uniform in $\log \sigma(\mathrm{C})$ for all model populations, with $-1.75<\log \sigma(\mathrm{C})<0$;

- uniform in $\left\langle C_{1.2-4}\right\rangle$ in the interval $0<\left\langle C_{1.2-4}\right\rangle<2$ for all model populations; uniform in $\log \sigma\left(C_{1.2-4}\right)$ in the interval $-3<\log \sigma\left(C_{1.2-4}\right)<-0.5$ for GCs and ICGCs and $-3<\log \sigma\left(C_{1.2-4}\right)<0.5$ for the extended contaminants.

\subsection{Completeness correction}

The mixture model just described allows us to infer the joint posterior distribution of the two dimensionless free parameters $f_{G C}$ and $R_{\mathrm{h}} / R_{S}$ which characterise each GCS. For each UDG, our final inference on the total abundance of the GCS, $N_{G C}$, is obtained by taking into account both spatial and magnitude incompleteness:

$N_{\mathrm{GC}}^{l}=N f_{G C}^{l} \times \frac{\int \Sigma\left(r, R_{\mathrm{h}}^{l}\right)}{\int S_{\mathrm{sp}}(\mathbf{r}) \Sigma\left(r, R_{\mathrm{h}}^{l}\right)} \frac{\int g_{G C}(F 814 W)}{\int S_{814} g_{G C}(F 814 W)}$,

where $N$ is the number of H10 sources in the UDG field, the index $l$ runs over our Markov chains, $S_{814}$ is the completeness function defined in Section 2.1 and $g_{G C}(F 814 W)$ is the GCLF of the member GCs. Since we will not be able to fully characterise $g_{G C}$ and because of the additional uncertainties introduced by the flux correction described in Section 2.1, we adopt a fixed Gaussian GCLF with parameters typical for dwarf galaxies: a mean of $\langle F 814 W\rangle=27.33 \mathrm{mag}$, and a spread of $\sigma_{F 814 W}=1.1 \mathrm{mag}$ (G10, Miller \& Lotz 2007; Peng et al. 2009). This implies a correction of a factor $\approx 2$. It is worth noting that the turnover and spread of the GCLF become respectively fainter and tighter in dwarf galaxies (e.g. Jordán et al. 2007). Therefore, our assumptions on the properties of $g_{G C}$ are conservative: using the GCLF of a bright galaxy would imply a smaller completeness correction, and therefore a lower GC richness for the same inferred value of $f_{G C}$. Over the entire sample of 54 UDGs, our analysis uses a total of 42703 distinct catalogue sources. 


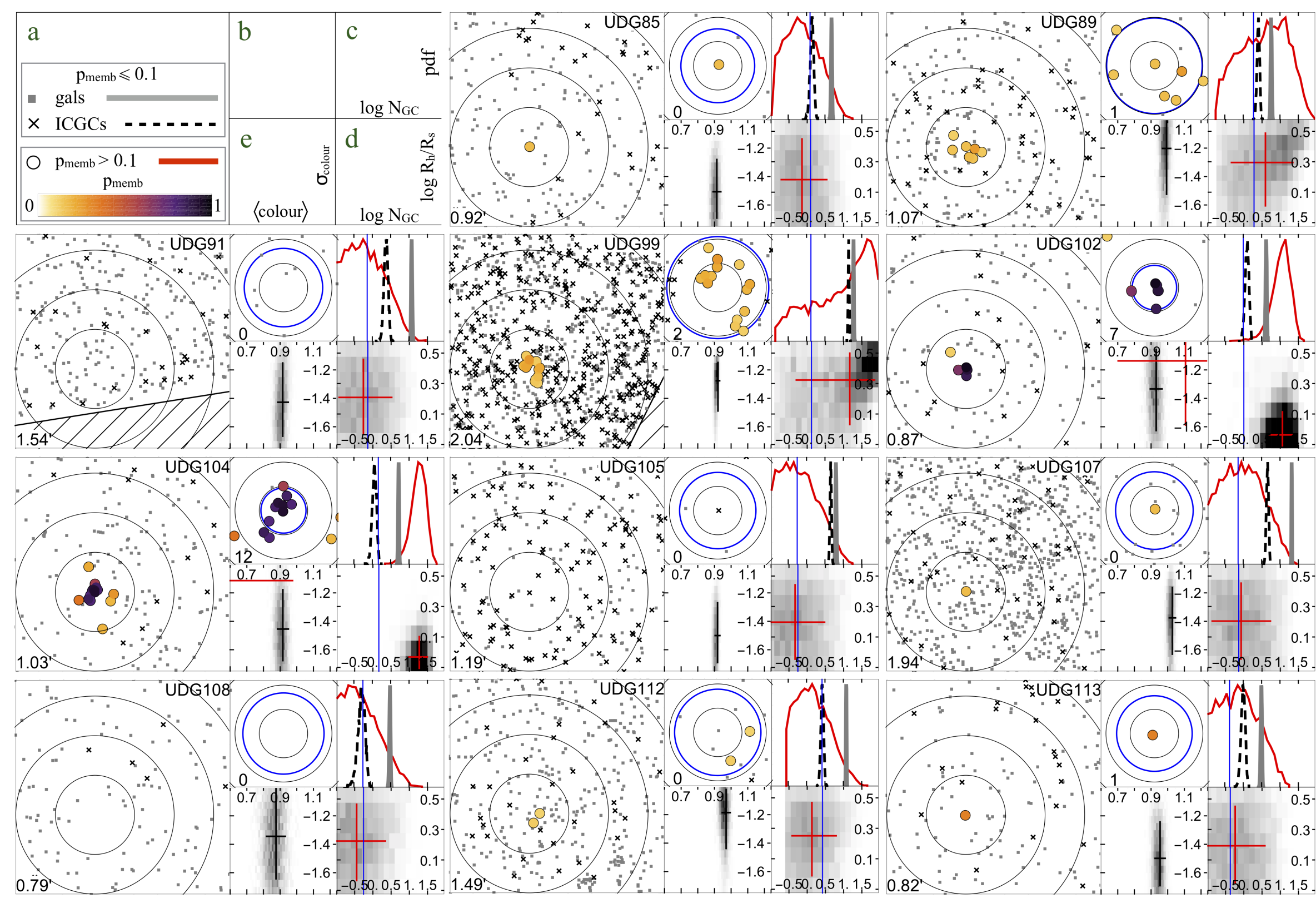




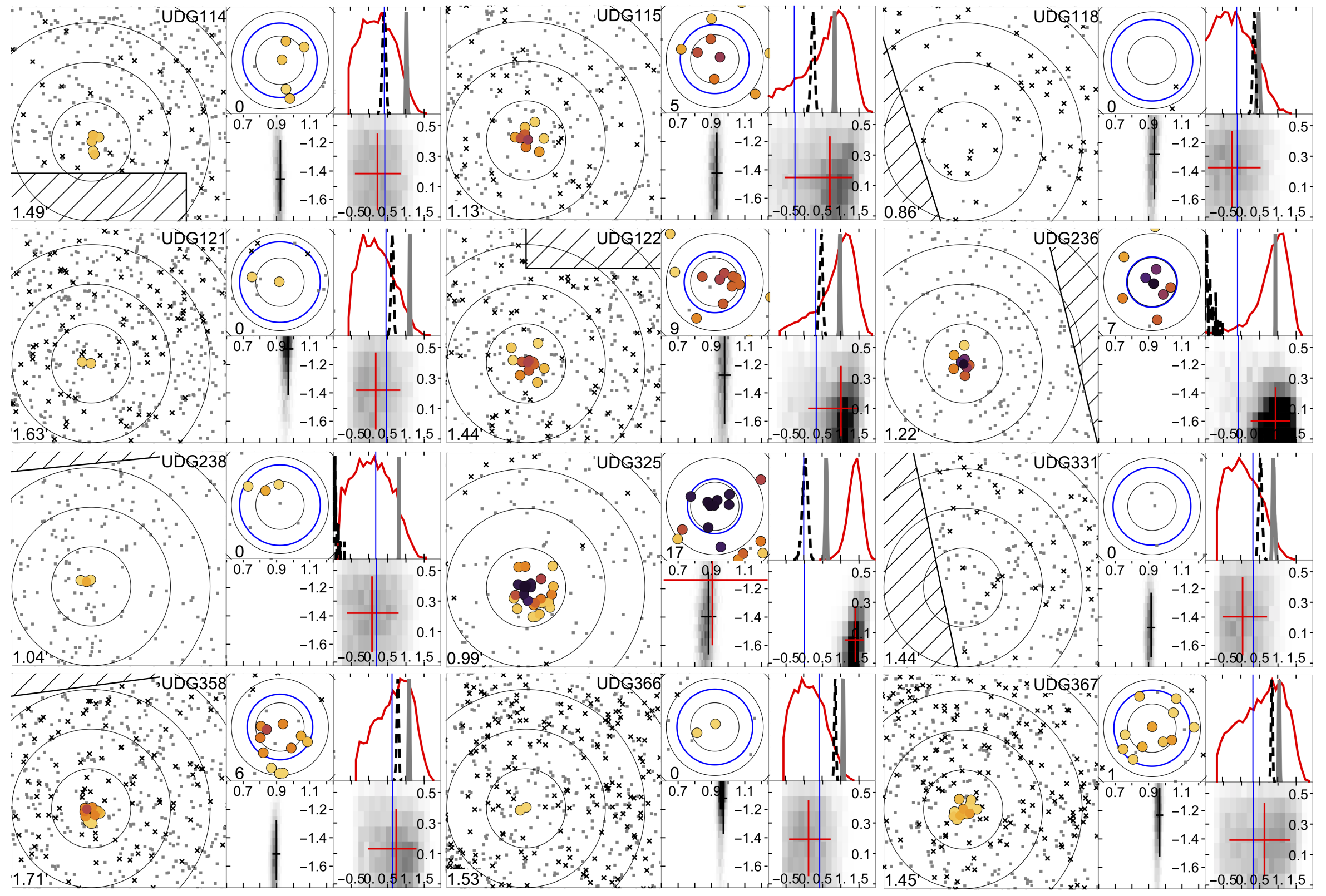




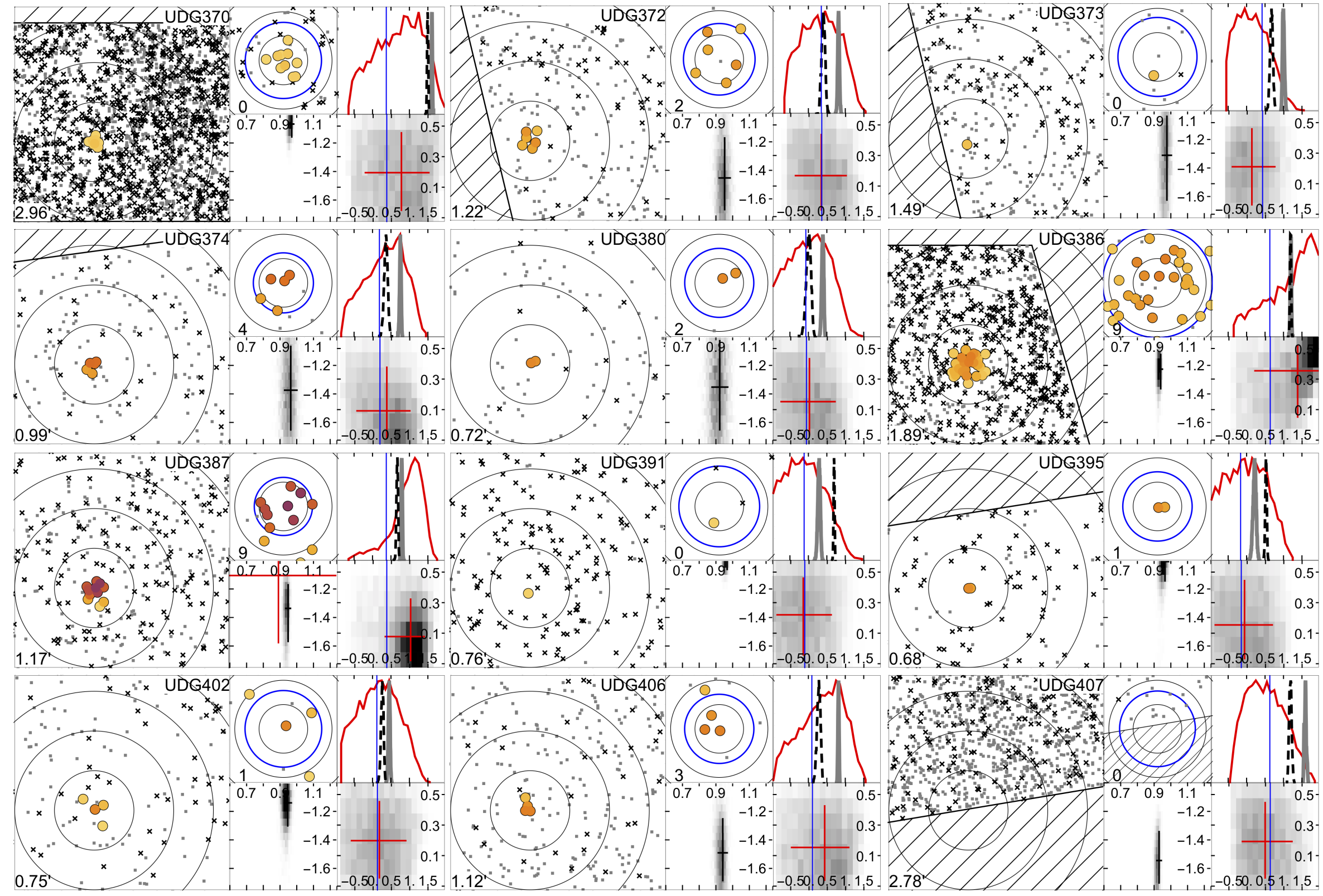



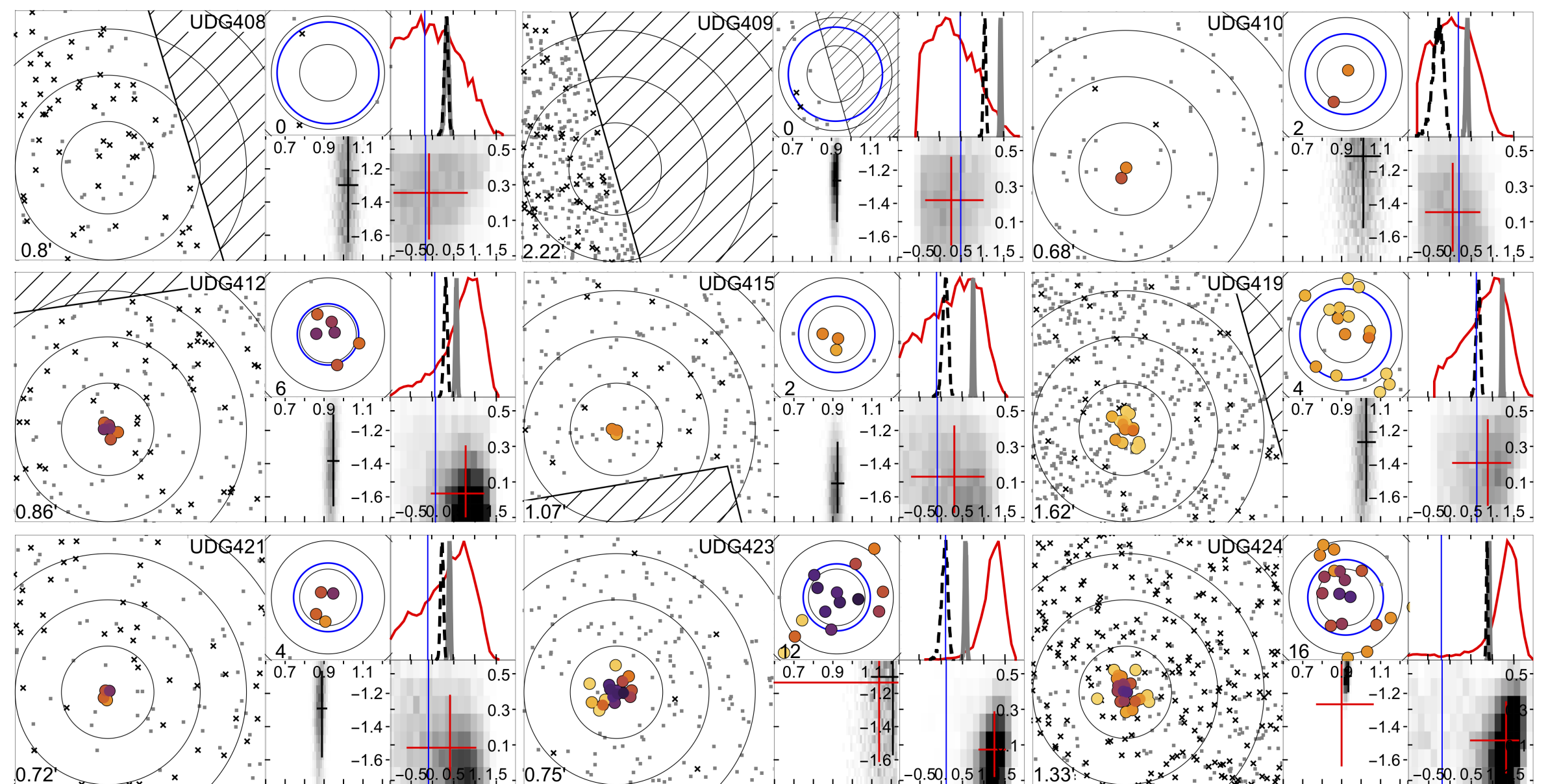

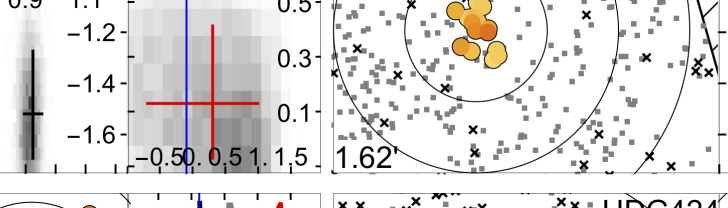
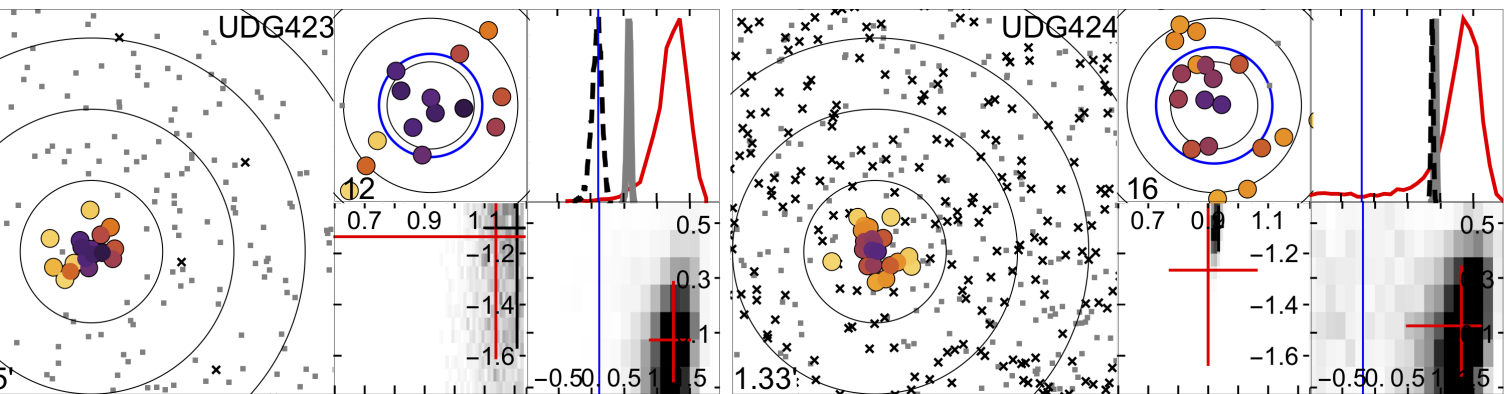

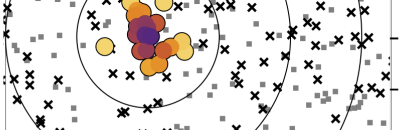

0.72

$1.6-0.50 .0 .51 \cdot 1,5 \cdot 0.75$
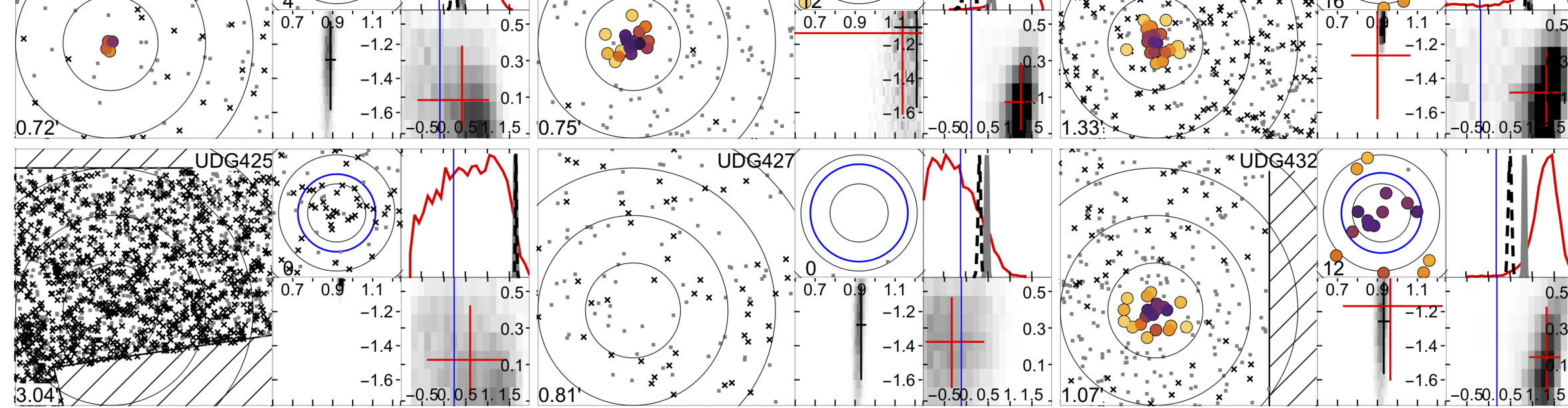


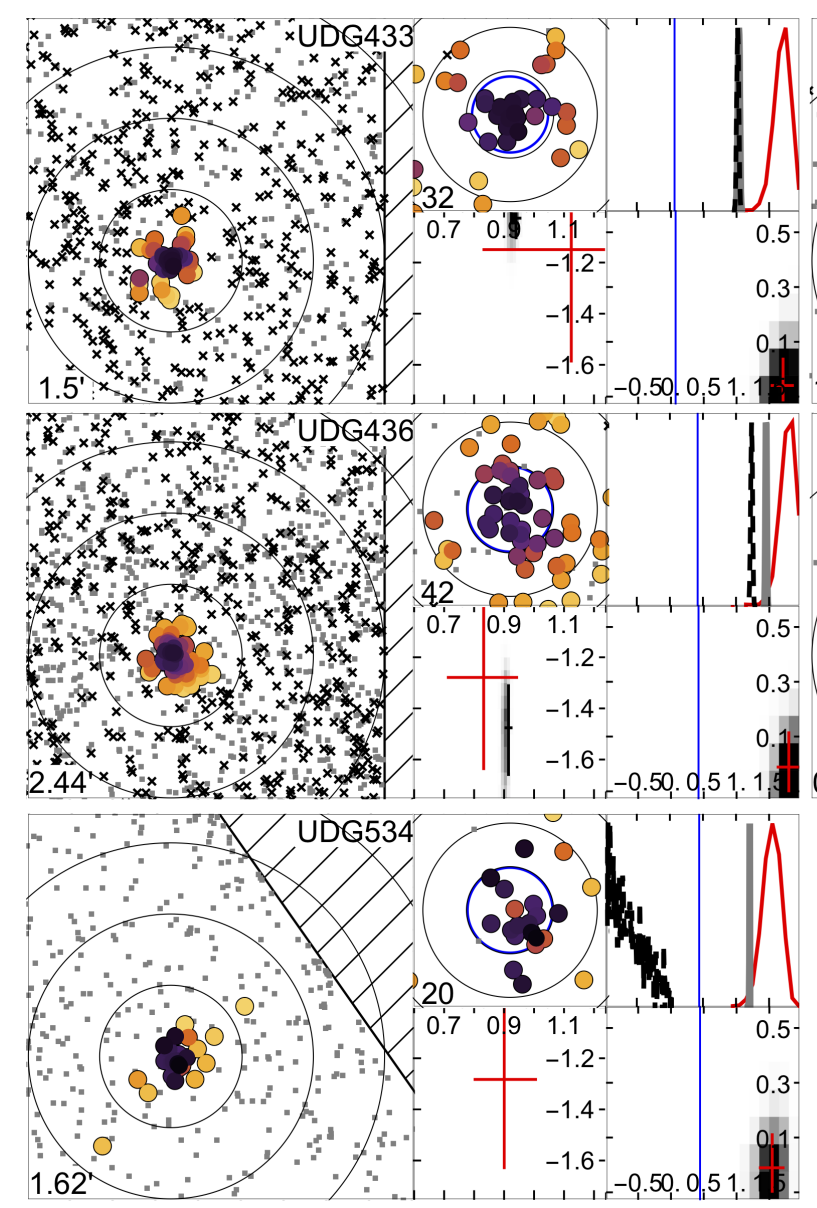

Figure 3. Results of the analyses on individual systems. The ID number of each UDG is in the upper-right of panels $a$. Panels $a$ and $b$ zoom on Fig. 1, around each UDG. Black concentric circles display $\{5,10,15,20\} \times R_{S}$ in panels $a$ and $\{1,2\} \times R_{S}$ in panels $b$, where $R_{S}$ is the stellar half-light radius of the UDG. Panels $a$ have a size $l \times l$, where $l$ is indicated in the lower-left of the panel itself, in arcmin. Where visible, the oblique black masking shows areas that lie outside the CCTp coverage and areas that are excluded due to bright galaxies. 作

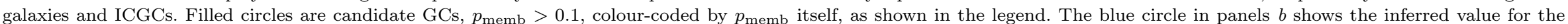
half-count radius of the UDG GC system. Panels $c$ and $d$ show our inference on the UDG GC abundance, $N_{G C}$, after correcting by spatial and magnitude incompleteness, in red. The integer in the lower-left of panels $b$ shows the number of GCCs with $p_{\text {memb }}>0.25$. Grey and black probability distribution functions in panels $c$ show the GC abundance that would be inferred as a result of the contaminant population of resolved galaxies and ICGCs, respectively. The vertical blue line in the same panels shows the mean GC abundance of normal dwarf galaxies with the same stellar mass, as from eqn. (10). Panels $e$ display inferences on the colour properties of the ICGCs (grey shading and black cross) and, where at least 5 GCCs with $p_{\text {memb }}>0.5$ are identified, for the member GCs (red cross). In both panels $d$ and $e$, crosses extend between the $10 \%$ and $90 \%$ quantiles of the relevant posterior distributions. 


\section{RESULTS}

Results for all our 54 UDGs are presented in Fig. 3 and Table 1, which collects $10 \%, 50 \%$ and $90 \%$ quantiles of the posterior distributions. Results for our hyper parameters are collected in Table 2. Fig. 3 shows a mosaic of 54 panels, dedicated to each of our 54 UDGs. Each panel is composed of insets $a$ to $e$, as indicated by the legend (first panel).

Insets $a$ display zooms of Fig. 1 which include the central regions of each UDG but are placed off-centre in order to allow a better impression of the statistics of the contaminants. Coordinates in these panels are scaled to the UDG's effective radius $R_{S}$, and the concentric black circles display $5,10,15$ and $20 \times R_{S}$, for scale. Panels $b$ focus on the innermost regions of the UDG, where members are concentrated when present. Black concentric circles in panels $b$ display 1 and $2 \times R_{S}$, while the blue circle shows our inference for the half-number radius of the UDG's GCS. In both panels $a$ and $b$, each catalogue source is displayed with a symbol that codes its probability of membership in the UDG GCS, $p_{\text {memb }}$, as obtained from our hierarchical Bayesian analysis. Sources that are contaminants with high probability $\left(p_{\text {memb }} \leqslant 10 \%\right)$ are displayed as a grey square or black cross based on whether they are more likely background galaxies or ICGCs. The remainder are shown as filled circles, colour-coded by their individual probability of membership, as shown by the legend. As mentioned earlier and clear in panels $a$ background counts are important in all studied systems. In the majority of cases, the number of background galaxies within $2 \times R_{S}$ is higher than the number of GCCs identified as high probability members. Only in a fraction of cases the distribution of sources shows obvious over-densities close to the centre of the UDG. The integer in the lower-left of insets $b$ displays the number of detected GCCs that have $p_{\text {memb }}>0.25$.

Insets $c$ display in red the marginalized posterior distribution for the richness of the GCS, $N_{G C}$, after correcting for both spatial and magnitude incompleteness, as in (9). For convenience, the probability distribution functions (pdfs) in these insets are normalised so that they peak at 1 . The vertical blue line shows the expected GC abundance based on the UDG stellar mass, $N_{G C, 0}$, according to the empirical relation

$\log N_{G C, 0}=0.58 \log \left(M^{*} / M_{\odot}\right)-4.09$,

which provides the best linear fit in log-log space to the sample of Georgiev et al. (2008, 2009, 2010). This collects $>50$ nearby dwarf galaxies with HST imaging (see Fig. 6). To enable use of the relation above, stellar masses for all our UDGs are obtained assuming that $B-R \sim 1$ for the UDGs (Koda et al. 2015), and averaging between the $M / L$ relations of Zibetti et al. (2009) (see their Table B1) and Bell et al. (2003) (see their Table 7). We return on the comparison between our inferred GC abundances and those that would be predicted by the empirical relation (10) in Section 4.2.

The grey-scale in insets $d$ shows the joint probability distribution for $N_{G C}$ and $\log R_{\mathrm{h}} / R_{S}$, with a red cross extending over the $10 \%$ and $90 \%$ quantiles of either posterior distributions. In most cases, the half-number radius $R_{\mathrm{h}}$ is undetermined. These correspond to the majority of cases in which there is no evidence for a central overdensity, and no high-probability candidate member is identified. In these instances, our inference on $N_{G C}$ is essentially an upper limit, while the inference on $R_{h}$ is mostly determined by the prior volume (see Appendix A). In turn, for a fraction of the UDGs, we clearly detect a system of member GCs, corresponding to well-defined bounds on both $N_{G C}$ and $\log R_{\mathrm{h}} / R_{S}$. It is worth noting that in the cases with apparently well detected GCSs, the preferred value of $R_{h}$ is often smaller than $R_{S}$ (see Table 1 ). This is even more striking considering that our prior for the ratio $R_{h} / R_{S}$ does not allow values $<0.75$ (or $\log R_{h} / R_{S}<-0.125$ ).

For comparison, panels $c$ also show marginalized posterior distribution for background galaxies (in grey) and ICGCs (black dashed). These report inferences for the quantities

$N_{I C G C}=N f_{I C G C} \times \frac{\int \Sigma\left(r, R_{\mathrm{h}} / R_{S}=2\right)}{\int S_{\mathrm{sp}}(\mathbf{r}) \Sigma\left(r, R_{\mathrm{h}} / R_{S}=2\right)} \mathcal{I}_{814}$,

$N_{\text {gal }}=N\left(1-f_{G C}-f_{I C G C}\right) \times \frac{\int \Sigma\left(r, R_{\mathrm{h}} / R_{S}=2\right)}{\int S_{\mathrm{sp}}(\mathbf{r}) \Sigma\left(r, R_{\mathrm{h}} / R_{S}=2\right)}$,

where $\mathcal{I}_{814}$ is the completeness correction

$\mathcal{I}_{814}=\frac{\int g_{G C}(F 814 W)}{\int S_{814} g_{G C}(F 814 W)}$.

$N_{I C G C}$ and $N_{\text {gal }}$ are therefore the number of members that would be inferred for that UDG as a consequence of the background counts in that field, statistically and assuming $R_{\mathrm{h}} / R_{S}=2$. In an analysis in which one would simply count sources in a given aperture around the UDG centre, the grey and black pdfs show inference for the contamination that should be subtracted. By comparing with the posterior for $N_{G C}$, it is clear that background counts are largely dominant in most systems, making this simple method to estimate GC abundances prone to significant uncertainties. Only those systems in which $N_{I C G C}+N_{\text {gal }}$ is significantly smaller than the inferred $N_{G C}$ do indeed display a significant overdensity associated with the UDG GCS.

Finally, panels $e$ address the colour properties of ICGCs and member GCs. It is worth noticing that, throughout the paper, results on the colour distribution of our populations of point sources - the population of member GCs and the ICGC population - take into account the filter dependence of the aperture correction, which we calculate using Sirianni et al. (2005), $\delta \mathrm{C}=0.09 \mathrm{mag}$. By comparing with the colour in a larger aperture, we estimate that resolved galaxies require a correction to their colour $\delta \mathrm{C}_{\text {gal }} \lesssim 0.01 \mathrm{mag}$, which we therefore decide not to apply in Table 2. For ICGCs and member GCs, inferences are displayed only when the number of members with probability $p>0.5$ in either populations is larger than 5 . The grey-scale shows the joint posterior distribution for the mean and dispersion of the colour of the ICGCs in each field, with the black cross ranging between the 10\%-90\% quantiles. Both mean colour and spread of the ICGCs vary from field to field, although they remain typical for GCs (see e.g., Peng et al. 2008; Peng \& Lim 2016; Beasley \& Trujillo 2016). Note that, the only fields in which we cannot constrain the colour properties of the ICGCs are UDGs 236, 238 and 534. As shown by Fig. 1, these galaxies lie in the CCTp fields that are further away from the centre of Coma, and therefore the surface density of ICGCs is expected to be lower there (Peng et al. 2011), in line with our 
findings. The fact we do not identify high probability members of the ICGC population in these fields implies that the number of foreground stars and background galaxies that 'contaminate' this population is negligible. The large majority of the high probability members of the ICGC population are indeed bona fide ICGCs. Unfortunately, we find it very difficult to constrain the colour properties of the different GCSs. The red crosses in panels $e$ show 10\%-90\% quantiles, but, even for those cases in which member GCs are securely identified, inferences remain rather inconclusive. We return on the colour properties of the member GCs in Section 4.3.

With reference to possible galactic nuclei, we consider the minimum distance of any high-probability members into the UDG GCS. When candidates with $p_{\text {memb }}>0.5$ exist within $150 \mathrm{pc}$ from the UDG centre, Table 1 records the minimum galactocentric distance $R_{\text {min }}$. There are 4 systems with high-probability members with $R_{\min } \leqslant 100 \mathrm{pc}$, and an additional 5 with $100<R_{\min } / \mathrm{pc}<150$.

\subsection{Upper limits for individual virial masses}

We follow Harris et al. (2015), and use GC abundance as a proxy for virial mass. In particular, we interpret value of the $90 \%$ quantile of the posterior distribution for $N_{G C}, N_{G C, 90}$ as an upper limit for the virial mass of each UDG, $M_{v i r, 90}$. In order to do so, we adopt the calibration presented by Harris et al. (2017), assuming its validity extends to the regime of UDGs:

$$
\left\{\begin{array}{l}
M_{\mathrm{vir}}=N_{G C} / \eta_{N} \\
\log \eta_{N}=-8.56-0.11 \log M_{v i r} / M_{\odot}
\end{array}\right.
$$

Results are listed in the second to last column of Table 1. In our sample of 54 systems, 33 galaxies have a virial mass $M_{v i r} \leqslant 10^{11} M_{\odot}$ at $90 \%$ probability. The remaining 21 are approximately equally split between systems with $M_{v i r, 90} \leqslant 4 \times 10^{11} M_{\odot}$ and systems with higher $90 \%$ upper limits. Note, however, that among these 21, 18 systems have GC abundances that are essentially undetermined because of the high background counts, with values of $M_{v i r, 10}$ below $10^{11} M_{\odot}$ also allowed. For only three galaxies we infer halo masses in excess of $10^{11} M_{\odot}$ at $90 \%$ probability. Interestingly, among the 21 galaxies with $M_{v i r, 90}>10^{11} M_{\odot}, 12$ have a half-light radius $R_{S}<1.5 \mathrm{kpc}$, implying that 9 of the 18 UDGs with $R_{S}>1.5 \mathrm{kpc}$ have $M_{v i r} \leqslant 10^{11} M_{\odot}$ at $90 \%$ probability.

\subsection{Comparison with 'normal' dwarfs}

Figure 4 illustrates how the GC abundances we measure compare with the values expected based on the UDG stellar masses. Coloured bars extend between the $10 \%$ and $90 \%$ quantiles of the quantity $N_{G C} / N_{G C, 0}$, where $N_{G C, 0}$ is according to the relation (10). UDGs are ordered (and colour-coded) by the stellar half-light radius $R_{S}$. Our results show that most galaxies in our sample have 'normal' GCSs for their stellar mass: the majority of the low surface brightness galaxies we can study are consistent the haloes of 'normal' dwarf galaxies. According to Fig. 4, 10 systems display GCSs that are 'overabundant' with respect to the mean relation at more than $90 \%$ probability; 9 galaxies have $N_{G C} / N_{G C, 0}>3$ at $90 \%$ probability. It is unclear, however,

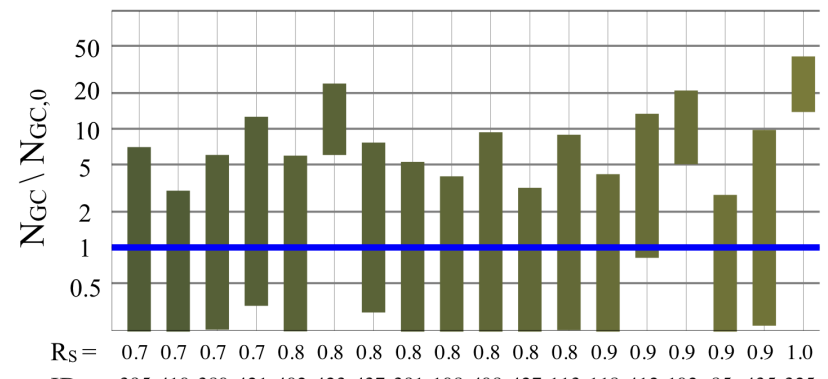

ID $=39541038042140242343739110840842711311841210285435325$

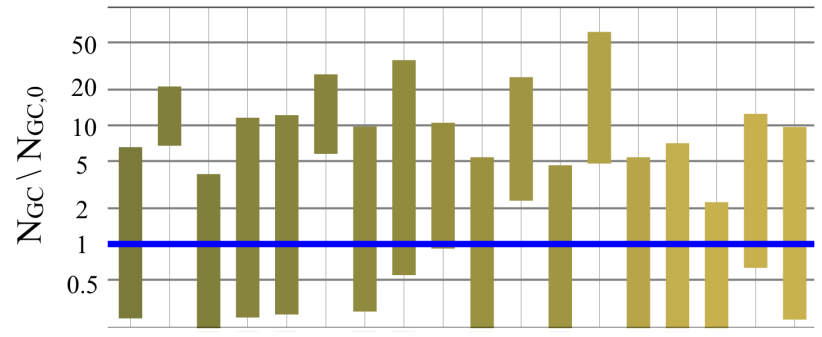

$\mathrm{R}_{\mathrm{S}}=\begin{array}{lllllllllllllllllllll}1.0 & 1.1 & 1.1 & 1.1 & 1.1 & 1.1 & 1.2 & 1.2 & 1.2 & 1.2 & 1.3 & 1.3 & 1.4 & 1.4 & 1.5 & 1.5 & 1.5 & 1.5\end{array}$ ID $=37410423889415432406115387105236372424434438331122367$

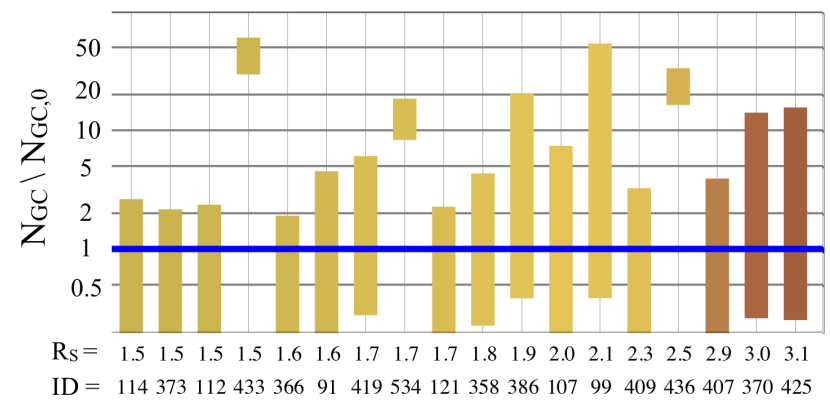

Figure 4. The richness of the UDG GC systems in terms of the mean richness of 'normal' dwarf galaxies with the same stellar mass, $N_{G C} / N_{G C, 0}$. The 54 UDGs in our sample are ordered by size, and colour-coded accordingly. Bars extend between the $10 \%$ and $90 \%$ quantiles.

whether this is particularly surprising given the substantial scatter about the relation (see Figure 6 below). We return on this aspect in Section 5. It is interesting to notice that while three of the 9 galaxies that satisfy $N_{G C, 10} / N_{G C, 0}>3$ have $R_{S}>1.5 \mathrm{kpc}$, the remaining 6 have smaller sizes, with 5 having $R_{S} \lesssim 1.1 \mathrm{kpc}$.

\subsection{The colour of member GCs}

As discussed in Section 4, the quality of the available data and the limited number of members do not, in most cases, allow for useful constraints on the colour properties of the individual UDG GCSs. We therefore perform a parallel analysis in which we assume that all of the UDG GCSs share the same mean colour $\langle\mathrm{C}\rangle_{G C}$ and dispersion $\sigma(\mathrm{C})_{G C}$, and elevate both to hyper parameters. This allows for improved constraints by using all of the detected high probability members over the 54 UDGs. We record our inference in Table 2 and display $10 \%$ to $90 \%$ quantiles of the joint probability distribution as a red cross in Figure 5. The same Figure collates results obtained for the same parameters for the ICGCs in each of the 54 studied fields, shown as thin black crosses. 
Table 1. Summary of the statistical constraints from our analyses. Column 1 lists the UDGs in our sample by their ID number, as in the catalogue by Yagi et al. (2016); columns 2 and 3 list stellar scale radii and stellar masses; column 4 collects the GC abundances expected based on the stellar mass of the system, according to eqn. (10); column 5 lists inferences on the GC abundance; column 6 records the ratio $R_{h} / R_{S}$; columns 7 and 8 refer to the properties of the colour distribution of the ICGCs in that field; column 9 uses inference on the GC abundance to produce upper limits (90\% quantiles) for the virial mass of each UDG, $M_{v i r, 90}$; when GC candidates with probability of membership into the UDG GCS $p_{\text {memb }}>0.5$ exist within $150 \mathrm{pc}$ from the UDG centre, column 10 lists the minimum galactocentric distance of these possible nuclei. Columns featuring a trio of entries collect the $\{10 \%, 50 \%, 90 \%\}$ of the relevant marginalised posterior distribution.

\begin{tabular}{|c|c|c|c|c|c|c|c|c|c|}
\hline ID & $\begin{array}{c}R_{S} \\
{[\mathrm{kpc}]}\end{array}$ & $\begin{array}{c}\log M_{*} \\
{\left[M_{\odot}\right]}\end{array}$ & $N_{G C, 0}$ & $N_{G C}$ & $\log R_{h} / R_{S}$ & $\begin{array}{c}\langle\text { colour }\rangle_{I C G C} \\
{[\mathrm{mag}]}\end{array}$ & $\begin{array}{c}\sigma\left(\text { colour }_{I C G C}\right) \\
{[\mathrm{mag}]}\end{array}$ & $\begin{array}{c}M_{\mathrm{vir}, 90} \\
{\left[10^{11} M_{\odot}\right]}\end{array}$ & $\begin{array}{l}R_{\min } \\
\mathrm{pc}\end{array}$ \\
\hline 85 & 0.94 & 7.18 & 1.2 & $\{0.2,0.7,3.4\}$ & $\{-0.06,0.18,0.45\}$ & $\{0.88,0.90,0.93\}$ & $\{0.02,0.03,0.05\}$ & 0.17 & - \\
\hline 89 & 1.10 & 7.50 & 1.9 & $\{0.5,3.9,22.1\}$ & $\{0.01,0.29,0.49\}$ & $\{0.96,0.98,1.01\}$ & $\{0.03,0.06,0.10\}$ & 1.3 & - \\
\hline 91 & 1.58 & 6.78 & 0.7 & $\{0.1,0.5,3.3\}$ & $\{-0.03,0.21,0.46\}$ & $\{0.88,0.91,0.94\}$ & $\{0.02,0.04,0.07\}$ & 0.16 & - \\
\hline 99 & 2.09 & 7.22 & 1.3 & $\{0.5,14.8,70.5\}$ & $\{0.03,0.32,0.50\}$ & $\{0.90,0.91,0.92\}$ & $\{0.03,0.05,0.07\}$ & 5.0 & - \\
\hline 102 & 0.89 & 7.02 & 1.0 & $\{5.0,11.5,21.0\}$ & $\{-0.11,-0.04,0.11\}$ & $\{0.89,0.92,0.96\}$ & $\{0.02,0.05,0.09\}$ & 1.3 & 122 \\
\hline 104 & 1.05 & 7.30 & 1.5 & $\{9.8,18.7,31.0\}$ & $\{-0.11,-0.03,0.10\}$ & $\{0.87,0.91,0.94\}$ & $\{0.02,0.04,0.07\}$ & 2.0 & 38 \\
\hline 105 & 1.22 & 6.58 & 0.6 & $\{0.1,0.5,3.0\}$ & $\{-0.05,0.20,0.44\}$ & $\{0.89,0.91,0.92\}$ & $\{0.02,0.03,0.05\}$ & 0.14 & - \\
\hline 107 & 1.99 & 6.78 & 0.7 & $\{0.1,0.8,5.4\}$ & $\{-0.04,0.20,0.45\}$ & $\{1.00,1.02,1.04\}$ & $\{0.02,0.04,0.07\}$ & 0.27 & - \\
\hline 108 & 0.81 & 6.58 & 0.6 & $\{0.1,0.4,2.2\}$ & $\{-0.04,0.22,0.47\}$ & $\{0.81,0.87,0.92\}$ & $\{0.02,0.05,0.10\}$ & 0.10 & - \\
\hline 112 & 1.53 & 7.74 & 2.6 & $\{0.4,1.3,6.2\}$ & $\{-0.01,0.25,0.47\}$ & $\{0.94,0.96,0.98\}$ & $\{0.04,0.07,0.09\}$ & 0.32 & - \\
\hline 113 & 0.84 & 6.38 & 0.4 & $\{0.1,0.6,3.8\}$ & $\{-0.05,0.19,0.45\}$ & $\{0.91,0.94,0.97\}$ & $\{0.02,0.03,0.06\}$ & 0.19 & - \\
\hline 114 & 1.52 & 7.74 & 2.6 & $\{0.4,1.7,6.9\}$ & $\{-0.05,0.19,0.44\}$ & $\{0.89,0.91,0.93\}$ & $\{0.02,0.03,0.07\}$ & 0.36 & - \\
\hline 115 & 1.16 & 6.58 & 0.6 & $\{0.3,5.0,19.7\}$ & $\{-0.06,0.15,0.42\}$ & $\{0.89,0.92,0.95\}$ & $\{0.02,0.04,0.07\}$ & 1.2 & - \\
\hline 118 & 0.88 & 6.82 & 0.8 & $\{0.1,0.6,3.2\}$ & $\{-0.03,0.23,0.46\}$ & $\{0.90,0.92,0.95\}$ & $\{0.03,0.05,0.09\}$ & 0.15 & - \\
\hline 121 & 1.67 & 7.82 & 2.9 & $\{0.5,1.5,6.6\}$ & $\{-0.03,0.22,0.46\}$ & $\{0.93,0.96,0.99\}$ & $\{0.04,0.08,0.12\}$ & 0.35 & - \\
\hline 122 & 1.47 & 7.58 & 2.1 & $\{1.3,10.1,26.5\}$ & $\{-0.08,0.10,0.38\}$ & $\{0.94,0.97,1.00\}$ & $\{0.02,0.05,0.10\}$ & 1.7 & - \\
\hline 236 & 1.25 & 6.86 & 0.8 & $\{1.9,8.6,20.6\}$ & $\{-0.10,0.02,0.23\}$ & - & - & 1.2 & 121 \\
\hline 238 & 1.06 & 7.34 & 1.5 & $\{0.3,1.2,6.0\}$ & $\{-0.03,0.22,0.46\}$ & - & - & 0.31 & - \\
\hline 325 & 1.01 & 7.02 & 1.0 & $\{13.9,25.0,40.8\}$ & $\{-0.09,0.05,0.27\}$ & $\{0.83,0.87,0.91\}$ & $\{0.02,0.04,0.08\}$ & 2.7 & 43 \\
\hline 331 & 1.47 & 7.58 & 2.1 & $\{0.3,1.1,4.8\}$ & $\{-0.04,0.21,0.46\}$ & $\{0.89,0.91,0.92\}$ & $\{0.02,0.03,0.06\}$ & 0.24 & - \\
\hline 358 & 1.75 & 8.10 & 4.3 & $\{1.0,5.4,18.4\}$ & $\{-0.06,0.13,0.39\}$ & $\{0.87,0.89,0.91\}$ & $\{0.02,0.03,0.05\}$ & 1.1 & - \\
\hline 366 & 1.57 & 7.74 & 2.6 & $\{0.4,1.3,5.0\}$ & $\{-0.04,0.19,0.44\}$ & $\{0.94,0.96,0.98\}$ & $\{0.04,0.08,0.10\}$ & 0.25 & - \\
\hline 367 & 1.48 & 7.58 & 2.1 & $\{0.5,4.3,20.5\}$ & $\{-0.04,0.20,0.43\}$ & $\{0.94,0.96,0.97\}$ & $\{0.03,0.06,0.09\}$ & 1.2 & - \\
\hline 370 & 3.03 & 7.66 & 2.4 & $\{0.6,5.9,33.3\}$ & $\{-0.05,0.20,0.46\}$ & $\{0.95,0.96,0.97\}$ & $\{0.07,0.09,0.10\}$ & 2.1 & - \\
\hline 372 & 1.25 & 7.62 & 2.2 & $\{0.4,2.2,10.3\}$ & $\{-0.06,0.17,0.44\}$ & $\{0.91,0.94,0.98\}$ & $\{0.02,0.04,0.07\}$ & 0.57 & - \\
\hline 373 & 1.52 & 7.74 & 2.6 & $\{0.4,1.3,5.7\}$ & $\{-0.04,0.21,0.46\}$ & $\{0.94,0.97,0.99\}$ & $\{0.02,0.05,0.09\}$ & 0.29 & - \\
\hline 374 & 1.01 & 7.34 & 1.5 & $\{0.4,2.4,10.1\}$ & $\{-0.08,0.09,0.38\}$ & $\{0.92,0.95,0.99\}$ & $\{0.02,0.04,0.09\}$ & 0.56 & - \\
\hline 380 & 0.74 & 6.90 & 0.9 & $\{0.2,1.0,5.1\}$ & $\{-0.06,0.15,0.43\}$ & $\{0.87,0.91,0.96\}$ & $\{0.02,0.04,0.09\}$ & 0.26 & - \\
\hline 386 & 1.93 & 8.10 & 4.3 & $\{1.6,24.0,86.6\}$ & $\{0.05,0.35,0.52\}$ & $\{0.92,0.93,0.94\}$ & $\{0.04,0.06,0.08\}$ & 6.2 & - \\
\hline 387 & 1.20 & 7.66 & 2.4 & $\{2.2,10.6,24.8\}$ & $\{-0.08,0.08,0.32\}$ & $\{0.92,0.94,0.95\}$ & $\{0.03,0.05,0.07\}$ & 1.5 & - \\
\hline 391 & 0.78 & 6.82 & 0.8 & $\{0.1,0.7,4.0\}$ & $\{-0.04,0.22,0.46\}$ & $\{0.94,0.96,0.97\}$ & $\{0.09,0.10,0.12\}$ & 0.20 & - \\
\hline 395 & 0.69 & 6.74 & 0.7 & $\{0.1,0.8,4.8\}$ & $\{-0.07,0.15,0.44\}$ & $\{0.94,0.96,0.98\}$ & $\{0.07,0.10,0.12\}$ & 0.24 & - \\
\hline 402 & 0.77 & 7.22 & 1.3 & $\{0.3,1.5,7.8\}$ & $\{-0.04,0.20,0.45\}$ & $\{0.91,0.94,0.96\}$ & $\{0.05,0.07,0.10\}$ & 0.42 & - \\
\hline 406 & 1.15 & 7.18 & 1.2 & $\{0.3,2.7,12.1\}$ & $\{-0.06,0.15,0.43\}$ & $\{0.90,0.93,0.96\}$ & $\{0.02,0.03,0.06\}$ & 0.69 & - \\
\hline 407 & 2.85 & 8.10 & 4.3 & $\{0.7,3.1,16.7\}$ & $\{-0.05,0.19,0.45\}$ & $\{0.91,0.92,0.93\}$ & $\{0.02,0.03,0.05\}$ & 0.98 & - \\
\hline 408 & 0.82 & 6.74 & 0.7 & $\{0.1,0.8,6.4\}$ & $\{-0.00,0.25,0.47\}$ & $\{0.97,1.01,1.06\}$ & $\{0.02,0.05,0.11\}$ & 0.34 & - \\
\hline 409 & 2.27 & 7.86 & 3.1 & $\{0.5,1.8,10.0\}$ & $\{-0.03,0.22,0.46\}$ & $\{0.90,0.92,0.94\}$ & $\{0.03,0.05,0.08\}$ & 0.55 & - \\
\hline 410 & 0.69 & 7.38 & 1.6 & $\{0.3,1.2,4.9\}$ & $\{-0.06,0.15,0.43\}$ & $\{0.91,1.00,1.09\}$ & $\{0.03,0.08,0.17\}$ & 0.25 & - \\
\hline 412 & 0.88 & 7.14 & 1.2 & $\{1.0,6.0,15.7\}$ & $\{-0.09,0.03,0.30\}$ & $\{0.91,0.94,0.96\}$ & $\{0.02,0.04,0.08\}$ & 0.92 & - \\
\hline 415 & 1.10 & 6.86 & 0.8 & $\{0.2,2.0,9.9\}$ & $\{-0.07,0.13,0.41\}$ & $\{0.89,0.91,0.94\}$ & $\{0.02,0.03,0.05\}$ & 0.54 & - \\
\hline 419 & 1.66 & 8.10 & 4.3 & $\{1.2,7.7,25.8\}$ & $\{-0.03,0.21,0.45\}$ & $\{0.97,1.02,1.06\}$ & $\{0.02,0.05,0.11\}$ & 1.6 & - \\
\hline 421 & 0.74 & 6.86 & 0.8 & $\{0.3,2.6,10.2\}$ & $\{-0.08,0.08,0.38\}$ & $\{0.86,0.88,0.90\}$ & $\{0.03,0.05,0.09\}$ & 0.56 & - \\
\hline 423 & 0.77 & 7.22 & 1.3 & $\{7.9,17.7,31.5\}$ & $\{-0.08,0.07,0.28\}$ & $\{1.10,1.20,1.30\}$ & $\{0.03,0.08,0.20\}$ & 2.0 & 131 \\
\hline 424 & 1.36 & 6.70 & 0.7 & $\{3.1,20.7,40.0\}$ & $\{-0.06,0.13,0.34\}$ & $\{0.91,0.92,0.94\}$ & $\{0.07,0.08,0.10\}$ & 2.6 & - \\
\hline 425 & 3.11 & 7.42 & 1.7 & $\{0.4,4.0,26.7\}$ & $\{-0.07,0.13,0.42\}$ & $\{0.93,0.94,0.95\}$ & $\{0.09,0.10,0.11\}$ & 1.7 & - \\
\hline 427 & 0.83 & 6.82 & 0.8 & $\{0.1,0.5,2.4\}$ & $\{-0.02,0.22,0.46\}$ & $\{0.90,0.92,0.94\}$ & $\{0.03,0.05,0.09\}$ & 0.11 & - \\
\hline 432 & 1.10 & 7.34 & 1.5 & $\{8.8,21.0,41.4\}$ & $\{-0.06,0.14,0.41\}$ & $\{0.90,0.92,0.95\}$ & $\{0.03,0.06,0.09\}$ & 2.7 & 52 \\
\hline 433 & 1.54 & 7.14 & 1.2 & $\{34.8,51.2,71.1\}$ & $\{-0.11,-0.06,0.04\}$ & $\{0.92,0.93,0.94\}$ & $\{0.08,0.09,0.11\}$ & 5.0 & 148 \\
\hline 434 & 1.38 & 7.42 & 1.7 & $\{0.3,1.8,9.2\}$ & $\{-0.07,0.13,0.41\}$ & $\{0.88,0.90,0.92\}$ & $\{0.02,0.03,0.05\}$ & 0.50 & - \\
\hline 435 & 0.95 & 6.74 & 0.7 & $\{0.2,1.1,6.7\}$ & $\{-0.04,0.19,0.44\}$ & $\{1.03,1.06,1.10\}$ & $\{0.02,0.04,0.08\}$ & 0.35 & 94 \\
\hline 436 & 2.50 & 7.74 & 2.6 & $\{43.0,62.6,87.6\}$ & $\{-0.10,-0.01,0.11\}$ & $\{0.90,0.90,0.91\}$ & $\{0.02,0.03,0.05\}$ & 6.3 & - \\
\hline 437 & 0.77 & 7.22 & 1.3 & $\{0.4,2.4,10.0\}$ & $\{-0.07,0.15,0.43\}$ & $\{0.85,0.91,0.96\}$ & $\{0.02,0.04,0.09\}$ & 0.55 & 141 \\
\hline 438 & 1.45 & 7.10 & 1.1 & $\{0.2,1.2,7.9\}$ & $\{-0.04,0.21,0.46\}$ & $\{0.89,0.90,0.92\}$ & $\{0.02,0.03,0.06\}$ & 0.42 & - \\
\hline 534 & 1.66 & 7.78 & 2.8 & $\{23.0,35.0,51.2\}$ & $\{-0.10,-0.01,0.11\}$ & - & - & 3.5 & - \\
\hline
\end{tabular}


Table 2. Inference on the model hyper-parameters.

\begin{tabular}{cc}
\hline$\left(\left\langle C_{1.2-4}\right\rangle, \sigma\left(C_{1.2-4}\right)\right)_{G C}$ & $(1.07,0.042) \mathrm{mag}$ \\
$\left(\left\langle C_{1.2-4}\right\rangle, \sigma\left(C_{1.2-4}\right)\right)_{\mathrm{gal}}$ & $(1.55,0.51) \mathrm{mag}$ \\
\hline$(\langle\mathrm{C}\rangle, \sigma(\mathrm{C}))_{G C}$ & $(0.91,0.06) \mathrm{mag}$ \\
$(\langle\mathrm{C}\rangle, \sigma(\mathrm{C}))_{I C G C}$ & $(0.93,0.07) \mathrm{mag}$ \\
$(\langle\mathrm{C}\rangle, \sigma(\mathrm{C}))_{\mathrm{gal}}$ & $(0.92,0.57) \mathrm{mag}$ \\
\hline
\end{tabular}

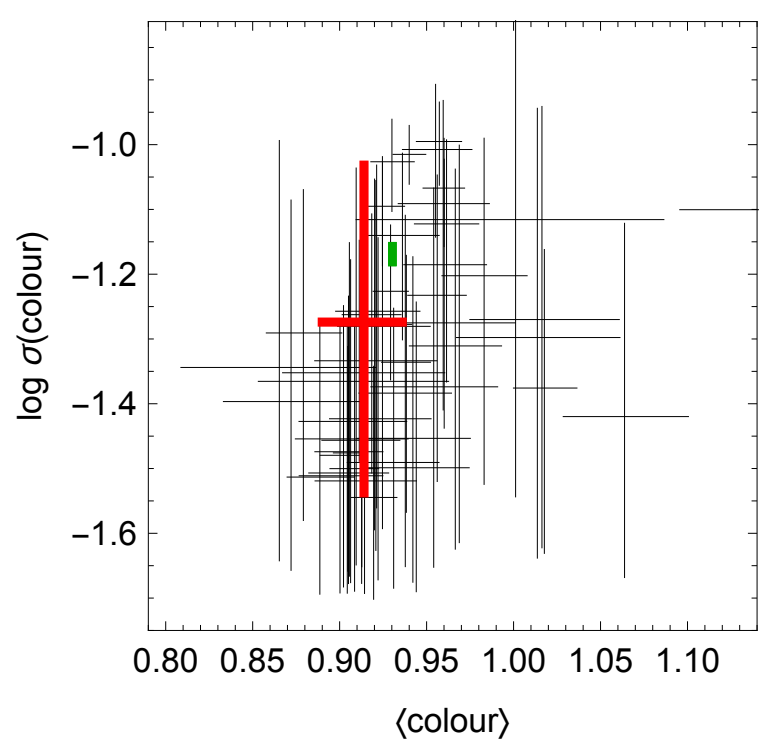

Figure 5. The colour properties inferred for the ensemble of UDG GCs (red cross, extending between $10 \%$ and $90 \%$ of the probability distribution for either mean colour and spread). These are compared with the properties of the ICGCs in each individual UDG field (thin black crosses), as well as with the properties of the ensemble of ICGCs in all studied fields (green rectangle, extending between $10 \%$ to $90 \%$ quantiles of the inferred probability distributions). Inferences are also reported in Table 2.

Most of these are perfectly compatible with what we find for the UDG GCSs. For a better comparison, we also elevate the properties of the ICGCs to hyper-parameters, to measure the mean properties of the ICGCs in all of Coma. Given the large number of ICGCs identified in our analysis, their properties are very well determined. We display results in Fig. 5 as a green rectangle, extending between $10 \%$ to $90 \%$ quantiles of the inferred probability distributions (mean values are appended in Table 2). Results obtained for the ensemble of the member GCs and for the ensemble of the ICGCs are perfectly compatible, in both mean colour and spread. The available data do not allow to distinguish the mean properties of the two populations. In other words, we cannot rule out that GC lost by disrupted UDGs make up for most of the ICGC population.

\subsection{Comparison with literature work}

Concurrently with this study, van Dokkum et al. (2017) (hereafter vD17) published an independent analysis that includes 12 of the galaxies in our sample, based on the same CCTp imaging used to produce the H10 catalogue. The parallel study by vD17, however, is different in that they perform their own source extraction, after explicitly fitting for and subtracting the 12 studied UDGs from the images themselves (rather than by correcting the aperture photometry after the source extraction as we do in Section 2.1). In addition, the vD17 analysis and the present one differ substantially in methodology, using two different techniques to assess GC abundances. While we perform a statistical analysis of all sources in large fields around each UDG, van Dokkum et al. (2017) adopts the approach of counting all sources within an aperture centred on the UDG to then subtract an estimate of the background contamination. This number count is then corrected for magnitude incompleteness (a factor of 2 , analogous to our factor $\mathcal{I}_{814}$ ) and by spatial incompleteness, assuming that all UDG GCSs have a halfnumber radius of $1.5 \times R_{S}$.

Our results are in rough agreement with those presented by vD17, with our uncertainties often wider than those recorded by that study. The most discrepant results are for UDGs with ID 358, for which vD17 records a lower 1-sigma limit of 31 while we find an upper limit of $N_{G C, 90}=18.4$, and ID 121, with respectively 14 and 6.6. In turn, we find that the richness of UDGs ID 122 and ID 386 have a high uncertainty because of the background counts, but could potentially be significantly higher than estimated by vD17.

\section{DISCUSSION AND CONCLUSIONS}

Our results show that the majority of UDGs are hosted by dwarf mass haloes, with $M_{v i r} \lesssim 10^{11} M_{\odot}$. For only 3 systems our inference on the richness of their GCS translates to a virial mass that is higher than $10^{11} M_{\odot}$ at $90 \%$ confidence (UDGs with ID 436, 433 and 534, have $M_{v i r, 10}>10^{11} M_{\odot}$ ). According to the bounds allowed by the currently available dataset, most UDG GCSs are in fact consistent with expectations for normal dwarf galaxies with the same stellar mass. A fraction of systems, however, display richer GCSs. This property does not seem to correlate with either stellar mass or galaxy size. We find 9 systems with GCS that are richer by more than 3 times (at $90 \%$ probability) with respect to the mean of the population of 'normal' dwarfs with similar stellar mass. Of these, three galaxies have $R_{S}>1.5 \mathrm{kpc}$, while the remaining six (UDGs with ID 102, 104, 325, 423, 424,432 ) are less extended, with half-light radii ranging between 0.8 and $1.4 \mathrm{kpc}$. These galaxies enlarge the sample of known dwarfs with especially abundant GCSs (see e.g., Lotz et al. 2004; Peng et al. 2008), adding nine low surface brightness systems.

Our results suggest that the extended size and unusually high GC abundance do not necessarily accompany each other. The physical mechanism responsible for the uncommonly high half-light radii of the UDGs has not yet been pinpointed unequivocally, but it appears that the 'over-abundance' of some UDG GCS may in fact be unrelated. In turn, this may call into question the threshold of $R_{S}>1.5 \mathrm{kpc}$ used so far to classify UDGs. Our sample of 54 low surface brightness galaxies includes extended UDGs $\left(R_{S}>1.5 \mathrm{kpc}\right)$ with normal GCSs and relatively compact dwarfs with clearly elevated GC abundances. Certainly, all of our galaxies are well within the dwarf regime, we find no single system where GC abundances typical for MW like galaxies $\left(N_{G C, M W} \approx 144\right.$, Harris et al. 2017) are allowed within the $90 \%$ confidence region. 


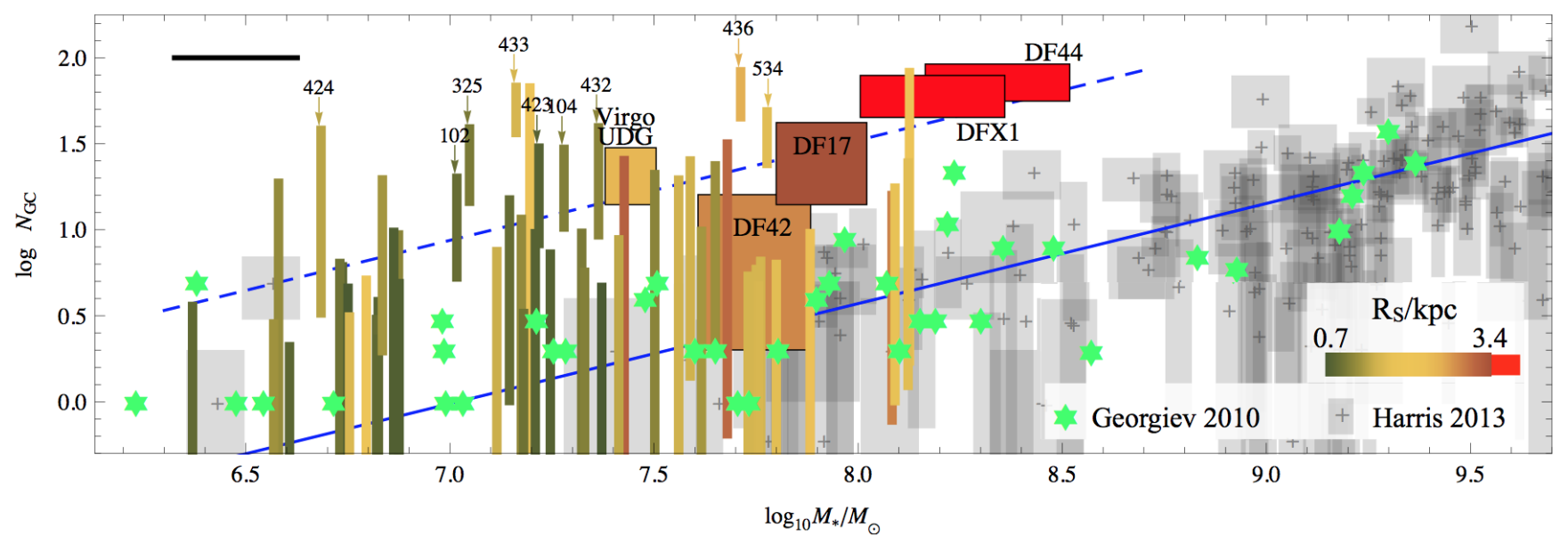

Figure 6. The richness of the GCS of 'normal' dwarf galaxies, from the H13 catalogue (grey rectangles) and from G10 (green stars). The solid blue line is a fit to the properties of the dwarf galaxies collected by Georgiev et al. (2008, 2009, 2010), recorded in eqn. (10). Results for all our 54 UDGs (90\% confidence regions) are shown as coloured bars, colour-coded by the UDG stellar half-light radius. The horizontal black bar in the upper-left illustrates the size of the uncertainty on the UDG stellar masses. Literature measurements for UDG GC abundances are shown as full rectangles with a black edge, with the same colour-coding. The dashed blue line provides fit to the relation that characterises galaxies with 'enhanced' GC richness with respect to their stellar mass, characterized by GC abundances approximately 9 times higher than normal galaxies.

In addition, it is worth noticing that, in those systems in which large values of $N_{G C} / N_{G C, 0}$ are securely identified, our analysis suggests that $R_{h} / R_{S} \lesssim 2$ with high probability, with at least 4 cases in which $R_{h} / R_{S} \lesssim 1$. Thus the GCSs in our objects have values of $R_{h}$ which resemble those of normal galaxies of the same stellar mass rather than of the same size. Under the hypothesis that GCS properties are more closely related to dark matter halos than to central galaxies, this may suggest that halo extent (and hence mass) is more closely linked to stellar mass than to size in our objects. This may provide independent evidence that these objects do not have overmassive haloes compared to the expectation for their stellar mass, though deeper datasets, to better account for the high background counts, would be valuable.

\subsection{A separate cluster population of GC-rich galaxies?}

Fig. 6, compares the inferred GC abundances of our 54 UDGs with those of nearby galaxies from the compilations of G10 (green stars) and Harris et al. (2013, H13, grey rectangles), as a function of stellar mass ${ }^{1}$. The blue solid line represents the relation (10), which, we recall, is a fit to the properties of the sample of nearby dwarf galaxies by Georgiev et al. $(2008,2009,2010)$. Note that this includes a minority of galaxies that are not displayed in Fig. 6, as bearing no GCs. Vertical bars (colour-coded by stellar half-light radius) cover the range of $\mathrm{GC}$ abundances that are compatible with the CCTp data at $90 \%$ confidence, while the horizontal black bar in the upper-left illustrates the size of the uncertainty

1 For the H13 catalogue, stellar masses were obtained using morphological type as a proxy for colour. For our UDGs, the uncertainty displayed in the top-left shows the difference between the masses inferred using the $M / L$ relations of Zibetti et al. (2009) and Bell et al. (2003). on the UDG stellar masses. Full rectangles display literature measurements for other UDGs not included in our sample (Peng \& Lim 2016; Beasley \& Trujillo 2016; van Dokkum et al. 2017).

Beasley \& Trujillo (2016) and Peng \& Lim (2016) have suggested that, though hosted by dwarf haloes with masses similar to that of the LMC, UDGs might still have rich GCSs for their stellar mass. This could be interpreted in two different ways: i) as a sign of 'failure' in forming stars, i.e. as an especially low star formation efficiency or as a consequence of premature gas removal or quenching of some form (van Dokkum et al. 2015; Yozin \& Bekki 2015; Beasley \& Trujillo 2016; Peng \& Lim 2016); ii) as the result of an especially high GC formation efficiency (e.g., Peng et al. 2008).

To address this, we perform a statistical analysis on the relation between stellar mass and GC abundance in our sample. We aim to establish whether the population of Coma low surface brightness galaxies, as probed by our 54 systems, can be described by a single population of galaxies (with significant scatter in the relation between stellar mass and GC abundance), or whether the data suggest the existence of an additional population with especially rich GCSs. We do so by describing our results in the $\left(M^{*}, N_{G C}\right)$ plane with a two component model, in which the first population is as from the relation (10) while a second population has GCSs richer by a factor $\mathrm{X}$ for the same stellar mass (and the same slope). Similarly to eqns. (5-7), UDG $j$ has a probability of belonging into the population of normal galaxies of

$$
\begin{aligned}
p_{\text {norm }, j}= & \frac{f_{\text {norm }}}{\sqrt{2 \pi\left[\sigma_{\text {norm }}^{2}+\sigma\left(\log N_{G C}\right)_{j}^{2}\right]}} \\
& \exp \left[-\frac{1}{2} \frac{\left(\left\langle\log N_{G C}\right\rangle_{j}-\log N_{G C, 0}\left(M_{j}^{*}\right)\right)^{2}}{\sigma_{\text {norm }}^{2}+\sigma\left(\log N_{G C}\right)_{j}^{2}}\right],
\end{aligned}
$$

and a probability of having an 'enhanced' GCS of

$$
\begin{aligned}
p_{\text {enh }, j}= & \frac{f_{\mathrm{enh}}}{\sqrt{2 \pi\left[\sigma_{\mathrm{enh}}^{2}+\sigma\left(\log N_{G C}\right)_{j}^{2}\right.}} \times \\
& \exp \left[-\frac{1}{2} \frac{\left(\left\langle\log N_{G C}\right\rangle_{j}-\log N_{G C, 0}\left(M_{j}^{*}\right)-\log X\right)^{2}}{\sigma_{\mathrm{enh}}^{2}+\sigma\left(\log N_{G C}\right)_{j}^{2}}\right],
\end{aligned}
$$


where $f_{\text {enh }}=1-f_{\text {norm }}$. The parameters of this model are:

- the fraction of normal galaxies, $f_{\text {norm; }}$;

- the logarithmic shift between the two abundance relations $\log X$;

- the intrinsic spreads of the two relations between stellar mass and GC abundance, for normal galaxies $\sigma_{\text {norm, }}$, and enhanced galaxies, $\sigma_{\text {enh }}$.

As the population of normal galaxies should describe the sample of G10, we impose a gaussian prior on $\sigma_{\text {norm }}$, with properties $\sigma_{\text {norm }}=0.40 \pm 0.05$. The likelihood of this model is

$\mathcal{L}=\prod_{j}^{54}\left(p_{\text {norm }, j}+p_{\text {enh }, j}\right)$.

Our 54 measured GC abundances suggest that a model with two distinct populations is preferred: the inferred mean for the parameter $\left\langle f_{\text {norm }}\right\rangle=0.7$ is about 3 -sigma away from $f_{\text {norm }}=1$, which identifies a model in which all galaxies are 'normal'. At the same time, the factor $X$ is estimated at $\langle X\rangle=9.2 \pm 1.9$, different from 1 with a similar significance. This shows that some galaxies in our sample are significant outliers from the relation (10). However, this does not necessarily imply that two distinct populations are needed to describe our sample. In fact, we find that a model featuring a single cluster population with common properties can describe our sample equally well. This requires a mean relation that is just slightly different than what prescribed by (10), and a wider intrinsic spread. The main cause for this is the large relative uncertainty on the majority of our inferred GC abundances.

The relation between stellar mass and the GCS richness of the enhanced galaxies is displayed by a blue dashed line in Fig. 6. Although data for DF44, DFX1, DF17 and the displayed Virgo UDG were not used in measuring the shift $X$, the resulting relation appears to provide a reasonable fit to their properties. In turn, despite its extended size, DF42 may in fact be best described by the relation for normal galaxies. Among our 54 low surface brightness galaxies, 9 are found to have a probability higher than $70 \%$ of belonging to the population of rich systems. These are highlighted with small arrows in Fig. 6 and have their ID numbers shown. In fact, these are the same as the 9 systems having a GCS at least 3 times richer than expected at $90 \%$ probability. As commented in Section 5, not all of these galaxies are extended; in fact the majority have $R_{S}<1.5 \mathrm{kpc}$. The physical mechanisms that make dwarf galaxies especially extended do not appear to be closely related to those that make some of them rich in GCs. Deeper datasets allowing better constraints for a larger set of both normal and low surface brightness dwarfs (inside and outside clusters) are necessary to unequivocally determine whether cluster galaxies with rich GCSs are indeed a separate galaxy population. This will help understand how GC abundance relates to galaxy size, stellar mass and morphology of galaxies and guide the identification of the mechanisms that are responsible for these properties.

\section{ACKNOWLEDGEMENTS}

NA and AM acknowledge stimulating discussions with Chervin Laporte. NA and AM thank Mike Beasley and Abraham Loeb for comments on an early version of this draft and are delighted to thank Pieter van Dokkum and Roberto Abraham for their useful insight during the development of this project and for sharing their results prior to publication.

\section{REFERENCES}

Amorisco, N. C., \& Evans, N. W. 2011, MNRAS, 411, 2118

Amorisco, N. C., Evans, N. W., \& van de Ven, G. 2014, Nat, 507, 335

Amorisco, N. C., \& Loeb, A. 2016, MNRAS, 459, L51

Beasley, M. A., Romanowsky, A. J., Pota, V., et al. 2016, ApJL, 819, L20

Beasley, M. A., \& Trujillo, I. 2016, ApJ, 830, 23

Behroozi, P. S., Wechsler, R. H., \& Conroy, C. 2013, ApJ, 770, 57

Bell, E. F., McIntosh, D. H., Katz, N., \& Weinberg, M. D. 2003, ApJS, 149, 289

Bellazzini, M., Belokurov, V., Magrini, L., et al. 2017, MNRAS,

Caldwell, N., \& Romanowsky, A. J. 2016, ApJ, 824, 42

Campbell, D. J. R., Frenk, C. S., Jenkins, A., et al. 2017, MNRAS, 469, 2335

Di Cintio, A., Brook, C. B., Dutton, A. A., et al. 2017, MNRAS, 466, L1

Forbes, D. A., Alabi, A., Romanowsky, A. J., et al. 2016, MNRAS, 458, L44

Gao, L., White, S. D. M., Jenkins, A., Stoehr, F., \& Springel, V. 2004, MNRAS, 355, 819

Georgiev, I. Y., Goudfrooij, P., Puzia, T. H., \& Hilker, M. 2008, AJ, 135, 1858-1876

Georgiev, I. Y., Puzia, T. H., Hilker, M., \& Goudfrooij, P. 2009, MNRAS, 392, 879

Georgiev, I. Y., Puzia, T. H., Goudfrooij, P., \& Hilker, M. 2010, MNRAS, 406, 1967

Giocoli, C., Tormen, G., \& van den Bosch, F. C. 2008, MNRAS, 386,2135

Greco, J. P., Greene, J. E., Price-Whelan, A. M., et al. 2017, arXiv:1704.06681

Guo, Q., White, S., Li, C., \& Boylan-Kolchin, M. 2010, MNRAS, 404, 1111

Hammer, D., Verdoes Kleijn, G., Hoyos, C., et al. 2010, ApJS, 191,143

Harris, W. E., Harris, G. L. H., \& Alessi, M. 2013, ApJ, 772, 82

Harris, W. E., Harris, G. L., \& Hudson, M. J. 2015, ApJ, 806, 36

Harris, W. E., Blakeslee, J. P., \& Harris, G. L. H. 2017, ApJ, 836, 67

Hudson, M. J., Harris, G. L., \& Harris, W. E. 2014, ApJL, 787, L5

Janssens, S., Abraham, R., Brodie, J., et al. 2017, ApJL, 839, L17

Jordán, A., McLaughlin, D. E., Côté, P., et al. 2007, ApJS, 171, 101

Kartha, S. S., Forbes, D. A., Spitler, L. R., et al. 2014, MNRAS, 437,273

Koda, J., Yagi, M., Yamanoi, H., \& Komiyama, Y. 2015, ApJL, 807, L2

Lee, M. G., Kang, J., Lee, J. H., \& Jang, I. S. 2017, ApJ, 844, 157

Leisman, L., Haynes, M. P., Janowiecki, S., et al. 2017, ApJ, 842, 133

Lotz, J. M., Miller, B. W., \& Ferguson, H. C. 2004, ApJ, 613, 262

Mackey, A. D., \& Gilmore, G. F. 2003, MNRAS, 338, 85 
Martínez-Delgado, D., Läsker, R., Sharina, M., et al. 2016, AJ, 151, 96

Gu, M., Conroy, C., Law, D., et al. 2017, arXiv:1709.07003

Mihos, J. C., Durrell, P. R., Ferrarese, L., et al. 2015, ApJL, 809, L21

Miller, B. W., \& Lotz, J. M. 2007, ApJ, 670, 1074

Moster, B. P., Naab, T., \& White, S. D. M. 2013, MNRAS, 428, 3121

Mowla, L., van Dokkum, P., Merritt, A., et al. 2017, arXiv: 1710.03762

Muñoz, R. P., Eigenthaler, P., Puzia, T. H., et al. 2015, ApJL, 813, L15

Peng, E. W., Jordán, A., Côté, P., et al. 2008, ApJ, 681, 197-224

Peng, E. W., Jordán, A., Blakeslee, J. P., et al. 2009, ApJ, 703, 42

Peng, E. W., Ferguson, H. C., Goudfrooij, P., et al. 2011, ApJ, 730, 23

Peng, E. W., \& Lim, S. 2016, ApJL, 822, L31

Plummer, H. C. 1911, MNRAS, 71, 460

Román, J., \& Trujillo, I. 2017, MNRAS, 468, 703

Salinas, R., Alabi, A., Richtler, T., \& Lane, R. R. 2015, AA, 577, A59

Schlafly, E. F., \& Finkbeiner, D. P. 2011, ApJ, 737, 103

Schlegel, D. J., Finkbeiner, D. P., \& Davis, M. 1998, ApJ, 500, 525

Shi, D. D., Zheng, X. Z., Zhao, H. B., et al. 2017, ApJ, 846, 26

Sifón, C., van der Burg, R. F. J., Hoekstra, H., Muzzin, A., \& Herbonnet, R. 2017, arXiv:1704.07847

Sirianni, M., Jee, M. J., Benítez, N., et al. 2005, PASP, 117, 1049

Trujillo, I., Román, J., Filho, M., \& Sánchez Almeida, J. 2017, arXiv:1701.03804

van der Burg, R. F. J., Muzzin, A., \& Hoekstra, H. 2016, AA, 590, A20

van der Burg, R. F. J., Hoekstra, H., Muzzin, A., et al. 2017, AA, 607, A79

van Dokkum, P. G., Abraham, R., Merritt, A., et al. 2015, ApJL, 798, L45

van Dokkum, P., Abraham, R., Brodie, J., et al. 2016, ApJL, 828, L6

van Dokkum, P., Abraham, R., Romanowsky, A. J., et al. 2017, ApJL, 844, L11

Venhola, A., Peletier, R., Laurikainen, E., et al. 2017, AA, 608, A142

Yagi, M., Koda, J., Komiyama, Y., \& Yamanoi, H. 2016, ApJS, 225,11

Yozin, C., \& Bekki, K. 2015, MNRAS, 452, 937

Walker, M. G., Mateo, M., Olszewski, E. W., et al. 2009, ApJ, 704, 1274

Walker, M. G., \& Peñarrubia, J. 2011, ApJ, 742, 20

Wolf, J., Martinez, G. D., Bullock, J. S., et al. 2010, MNRAS, 406,1220

Zaritsky, D., Crnojević, D., \& Sand, D. J. 2016, ApJL, 826, L9

Zaritsky, D. 2017, MNRAS, 464, L110

Zibetti, S., Charlot, S., \& Rix, H.-W. 2009, MNRAS, 400, 1181

\section{APPENDIX A: TEST SUITES}

Here we test our statistical framework, using purposely generated mock UDG datasets. We wish to evaluate our method's performance when applied to data with the properties of the CCTp data, especially with reference to the high background counts. Additionally, we wish to determine the prior distributions that are most appropriate for this study, exploring any biases they might give rise to.

\section{A1 Mock datasets}

For each of our 54 UDGs, we generate a set of 100 mock datasets with the same footprint as the real data, and therefore the same spatial selection function. Mock datasets comprise a population of member GCs, centred on the UDG, and a population of contaminants, which is distributed uniformly. For each UDG $j$, the contaminants' surface density $\Sigma_{c, j}$ is estimated using the real data, assuming that all catalogue sources are contaminants. This is an overestimate, but given that source counts are indeed largely dominated by the contaminants (see Fig. 3), its relative error remains negligible. For these tests, we do not distinguish between contaminants that appear as point sources and those that are extended, and are mainly concerned as to whether the richness of the GCS can be correctly inferred despite the strong background counts. We however seek to test whether source magnitudes can be used to help disentangle members. Therefore, a magnitude value for each contaminant source is generated by sampling randomly from the magnitude distribution of the $\mathrm{H} 10$ catalogue, $g_{\mathrm{H} 10}$, using again the fact that member GCs are only a very small fraction.

The mock member GCs are generated using the following model.

- The total richness of the GCS, $N_{G C}$, is generated assuming the galaxy is 'normal', meaning that it complies with the relation (10). For each of the 100 mock datasets, the value of $N_{G C}$ is therefore sampled from a Poisson distribution around the mean value prescribed by the relation (10) and the UDG's measured stellar mass (adopted values are listed in Table 1).

- The GCS has a Plummer or an exponential spatial distribution, each with a probability $p=0.5$. In our inference we assume a Plummer distribution: this allows us to test how the uncertainty on the actual profile of the UDG GCS affects our results. For either Plummer or exponential profiles, the half-count radius $R_{\mathrm{h}}$ has a Gaussian distribution with mean $R_{\mathrm{h}} / R_{S}=1.8$ and a scatter of 0.3 . This encompasses the properties of normal galaxies (e.g., Kartha et al. 2014; Caldwell \& Romanowsky 2016), as well as of UDGs studied so far (Beasley et al. 2016; Peng \& Lim 2016; van Dokkum et al. 2016).

- A magnitude value for each member GC is generated assuming the GCS has a Gaussian luminosity function, with values typical for normal dwarf galaxies (G10, Miller \& Lotz 2007; Peng et al. 2009). Different mock datasets, however, have different GCLF: individual turnovers and spreads are centred respectively in $\langle F 814 W\rangle=27.33 \mathrm{mag}$ and $\sigma_{F 814 W}=1.1 \mathrm{mag}$, and have a Gaussian distribution around these values with a scatter of 0.1 mag. Our completeness correction, eqn (9), assumes $\langle F 814 W\rangle=27.33$ and $\sigma_{F 814 W}=1.1$. As for the density profile of the GCS, this allows us to explore the effect that the uncertainty on the UDG GCLF has on our measurements.

The populations of both contaminants and members are filtered by the same spatial and magnitude selections that characterise the CCTp data. For each UDG, spatial selection excises all those mock GCCs that fall outside the available footprint, or in excised areas. Magnitude selection excises with probability $p=1-S_{814}(m)$ mock candidates with $F 814 W$ magnitude $m$. 


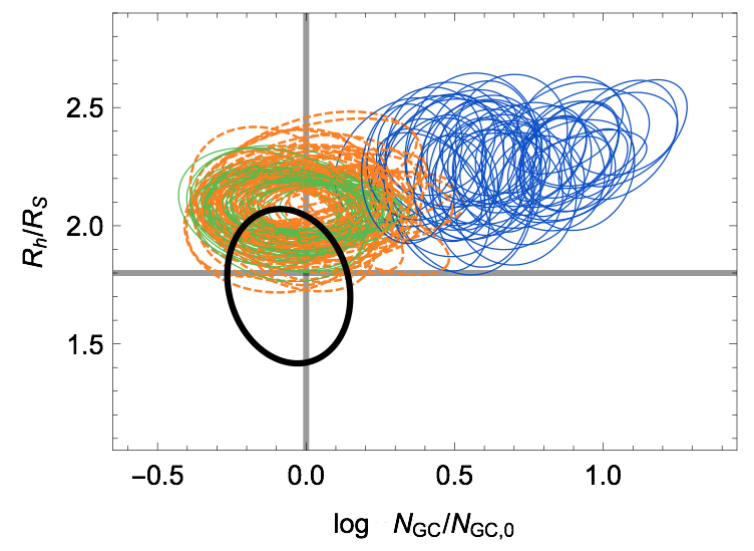

Figure A1. Performance of our statistical framework. Horizontal and vertical lines indicate the mean input parameters used to generate the mock datasets (a set of 100 for each UDG). For each UDG, coloured ellipses show the 1-sigma regions for the distributions of measurements on mock data (median GC abundance $N_{G C}$, median half-count radius $R_{\mathrm{h}} / R_{S}$ ). The mismatch between ellipses and lines quantifies bias. Solid ellipses correspond to results obtained without using the source magnitudes: in blue when adopting a prior with uniform density in $f$ and $R_{h} / R_{S}$, in green if the prior has uniform density in $\log f$ and $R_{h} / R_{S}$, in black for our final priors, with uniform density in $\log f$ and $\log R_{h} / R_{S}$. Orange dashed ellipses refer to results obtained using the source magnitudes (prior with uniform density in $\log f$ and $R_{h} / R_{S}$ ).

\section{A2 Prior distribution for the fraction $f$}

As already mentioned, the main difficulty presented by the data at hand lies in the dominant background counts. This implies that use of prior distributions that weigh differently the parameter volume $0<f_{G C}<1$ can influence our inferences. We isolate this problem here by testing a simplified mixture model that only uses the spatial distribution to disentangle members and contaminants:

$\mathcal{L}_{j}=\prod_{i}^{N_{j}}\left(p_{G C, i}+p_{\mathrm{cont}, i}\right)$,

where

$p_{G C, i}=f_{G C, j} \frac{S_{\mathrm{sp}, j}\left(\mathbf{r}_{\mathbf{i}}\right) \Sigma_{j}\left(r_{i}\right)}{\int S_{\mathrm{sp}, j} \Sigma_{j}(r)}$

$p_{\mathrm{cont}, i}=\left(1-f_{G C, j}\right) \frac{S_{\mathrm{sp}, j}\left(\mathbf{r}_{\mathbf{i}}\right)}{\int S_{\mathrm{sp}, j}}$,

and experiment with different prior distributions for the dimensionless free parameter $f_{G C, j}$. In these tests we put ourselves in the disadvantageous position of not using the colours or the concentration of our test sources. This reduces our power to disentangle members GCs from contaminants, which are differentiated by their spatial distribution alone. As a consequence, here we intend that the contaminant population include both resolved background galaxies and the ICGC population.

For instance, we consider a uniform prior in $f, 0<f<$ 1 , and a uniform prior in $\log f$. In the latter case, a finite lower bound is needed, and we use $\log f_{0}-1.5<\log f<0$, where the value $f_{0}$ is the fraction of members expected in the data when $N_{G C}=N_{G C, 0}$, i.e. if the GCS is 'normal', as in eqn. (10).
We first test the performance of the two different prior distributions, on all our 54 UDGs, using 100 mock datasets for each them. For each UDG mock dataset, we record results for the median values of the posterior distributions for both $R_{\mathrm{h}} / R_{S}$ and $N_{G C}$, after correcting the latter for completeness as in eqn (9). Hence, each mock dataset defines a point in this plane. Figure A1 displays the 1-sigma ellipse for the collection of these 100 points, one ellipse for each of our UDGs. Blue ellipses use the prior distribution with uniform density in $f$, green ellipses refer to the prior distribution with uniform density in $\log f$. The horizontal and vertical grey lines display the mean values of the input parameters used to generate the mock datasets: any systematic displacement of the ellipses quantifies bias. It is clear that the prior with uniform density in $f$ leads to a significant bias in both free parameters: GC abundances are significantly overestimated, by a factor $\gtrsim 3$. This is significantly ameliorated by adopting the prior distribution with uniform density in $\log f$, for which all 54 ellipses correctly include the input value for $N_{G C}$ within 1-sigma.

Some residual bias towards higher values is still present in our inference for the ratio $R_{\mathrm{h}} / R_{S}$, for which these tests assume a uniform prior $0.5<R_{\mathrm{h}} / R_{S}<3.5$. To correct for this bias, similarly to what done for the fraction $f$, we adopt a prior that is uniformly distributed in $\log R_{\mathrm{h}} / R_{S}$, in the interval $0.75<R_{\mathrm{h}} / R_{S}<3.5$. The black ellipse in Fig. A1 shows that this choice is appropriate.

\section{A3 Using the sources magnitude}

We also wish to test whether the additional magnitude information of each source might help disentangle members from contaminants. To do so, we update the model at eqns. $(\mathrm{A} 2, \mathrm{~A} 3)$ as follows:

$p_{G C, i}^{\prime}=p_{G C, i} \times \frac{\mathcal{G}_{\mathrm{GCLF}}\left(m_{i}\right) S_{814}\left(m_{i}\right)}{\int \mathcal{G}_{\mathrm{GCLF}} S_{814}}$,

$p_{\text {cont }, i}^{\prime}=p_{\text {cont }, i} \times g_{\mathrm{H} 10}\left(m_{i}\right)$,

where, $\mathcal{G}_{\mathrm{GCLF}}$ is the same GCLF adopted in producing the mock datasets and in performing our incompleteness correction (9), $S_{814}$ is the completeness function described in Section 2.1, while $g_{\mathrm{H} 10}$ is the magnitude distribution of the contaminants obtained as in $\mathrm{A} 1$, normalized to $\int g_{\mathrm{H} 10}=1$.

Results obtained using this model (and the prior with uniform density in $\log f$ ) are shown by orange dashed ellipses in Fig. A1. These do not show systematic improvement with respect to the simpler model of eqns. (A2,A3): the magnitude distributions of member GCs and contaminants are not sufficiently different from each other to guarantee a measurable statistical improvement. As a consequence, we do not use explicitly the magnitude of each source in our analysis on the real data. 